\title{
ON THE HARDY SPACE THEORY OF COMPENSATED COMPACTNESS QUANTITIES
}

\author{
SAULI LINDBERG
}

\begin{abstract}
We make progress on a problem of R. Coifman, P.-L. Lions, Y. Meyer, and S. Semmes from 1993 by showing that the Jacobian operator $J$ does not map $W^{1, n}\left(\mathbb{R}^{n}, \mathbb{R}^{n}\right)$ onto the Hardy space $\mathcal{H}^{1}\left(\mathbb{R}^{n}\right)$ for any $n \geq 2$. The related question about surjectivity of $J: \dot{W}^{1, n}\left(\mathbb{R}^{n}, \mathbb{R}^{n}\right) \rightarrow \mathcal{H}^{1}\left(\mathbb{R}^{n}\right)$ is still open.

The second main result and its variants reduce the proof of $\mathcal{H}^{1}$ regularity of a large class of compensated compactness quantities to an integration by parts or easy arithmetic, and applications are presented. Furthermore, we exhibit a class of nonlinear partial differential operators in which weak sequential continuity is a strictly stronger condition than $\mathcal{H}^{1}$ regularity, shedding light on another problem of Coifman, Lions, Meyer, and Semmes.
\end{abstract}

\section{INTRODUCTION}

Compensated compactness refers to the phenomenon of a nonlinear quantity enjoying nontrivial compactness or continuity properties due to, e.g., cancellations inherent in its algebraic structure. As an example relevant to this article, if $u^{j} \rightarrow u$ in $W^{1, n}\left(\mathbb{R}^{n}, \mathbb{R}^{n}\right)$, then the Jacobian determinants satisfy $J u^{j} \rightarrow J u$ in $\mathcal{D}^{\prime}\left(\mathbb{R}^{n}\right)$ even though the individual terms of the determinants need not converge in $\mathcal{D}^{\prime}\left(\mathbb{R}^{n}\right)$. Compensated compactness theory originated in the pioneering work of F. Murat and L. Tartar in the 1970's, and it has been used especially in the study of hyperbolic conservation laws. For information on compensated compactness see e.g. [8, [9], [10, 11], 19], 32, 35, 36, 433, 44, and the references contained therein.

The aim of this article is to advance the Hardy space theory of compensated compactness quantities which was initiated by R. Coifman, P.-L. Lions, Y. Meyer, and S. Semmes in the celebrated article 44. As shown in 4, numerous compensated compactness quantities such as Jacobians of mappings in $W^{1, n}\left(\mathbb{R}^{n}, \mathbb{R}^{n}\right)$

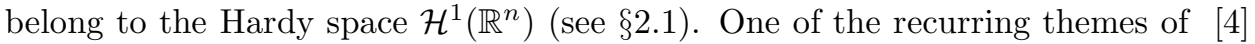
is the determination of the exact ranges of $\mathcal{H}^{1}$ regular compensated compactness quantities. In particular, the following problem is posed at [4, p. 258].

Question 1.1 ( 4]). Does the Jacobian operator map $W^{1,2}\left(\mathbb{R}^{2}, \mathbb{R}^{2}\right)$ onto $\mathcal{H}^{1}\left(\mathbb{R}^{2}\right)$ ?

This article provides a negative answer to Question 1.1 (see Theorem 1.2 and Corollary 1.3). We also present two general non-surjectivity results, Theorems 1.4 and 6.2, that cover various other quantities. The surjectivity question is, however, still open for operators whose domain of definition is an homogeneous Sobolev space, e.g. $J: \dot{W}^{1, n}\left(\mathbb{R}^{n}, \mathbb{R}^{n}\right) \rightarrow \mathcal{H}^{1}\left(\mathbb{R}^{n}\right)$ (see 97 ).

2010 Mathematics Subject Classification. Primary 46E30, 46N20; Secondary 35B45, 35A01.

The author was supported by the ERC Starting grant no. 307179. He also wishes to thank . 
Another question posed in 4] is whether compensated compactness and $\mathcal{H}^{1}$ regularity are, under natural conditions, equivalent - see $\$ 5.3$ for the exact statement of the problem in 4]. For a large and natural class of multilinear partial differential operators the answer is positive, as follows in an implicit way already from 44, Theorem V.1] and the work of R. Coifman and L. Grafakos in [5] and [15]. We give a rather elementary proof of one version of this fact and formulate the result in Corollary 5.12. In 86.2 we show that for more general homogeneous polynomials of partial derivatives, $\mathcal{H}^{1}$ regularity is a strictly weaker condition than compensated compactness.

As a partial result concerning Question 1.1. Coifman, Lions, Meyer, and Semmes showed that $\mathcal{H}^{1}\left(\mathbb{R}^{n}\right)$ is the minimal closed subspace of $\mathcal{H}^{1}\left(\mathbb{R}^{n}\right)$ that contains $J u$ for every $u \in W^{1, n}\left(\mathbb{R}^{n}, \mathbb{R}^{n}\right)$. They achieved this by proving the following analogue of the classical atomic decomposition of $\mathcal{H}^{1}\left(\mathbb{R}^{n}\right)$ : every $h \in \mathcal{H}^{1}\left(\mathbb{R}^{n}\right)$ can be expressed as $h=\sum_{j=1}^{\infty} \lambda^{j} J u^{j}$, where $\sum_{j=1}^{\infty}\left|\lambda^{j}\right| \lesssim\|h\|_{\mathcal{H}^{1}}$ and each $u^{j} \in W^{1, n}\left(\mathbb{R}^{n}, \mathbb{R}^{n}\right)$ satisfies $\left.\left\|\mathrm{D} u^{j}\right\|_{L^{n}} \leq 1\right]$ We show that the condition $\left\|\mathrm{D} u^{j}\right\|_{L^{n}} \leq 1$ cannot be replaced by $\left\|u^{j}\right\|_{W^{1, n}} \leq 1$.

Theorem 1.2. For every $n \geq 2$, the set

$$
\left\{\sum_{j=1}^{\infty} \lambda^{j} J u^{j}:\left\|u^{j}\right\|_{W^{1, n}} \leq 1 \text { for every } j \in \mathbb{N}, \sum_{j=1}^{\infty}\left|\lambda^{j}\right|<\infty\right\}
$$

is of the first category in $\mathcal{H}^{1}\left(\mathbb{R}^{n}\right)$.

Since the Jacobian determinant is $n$-homogeneous, Theorem 1.2 can be stated equivalently by saying that $\left\{\sum_{j=1}^{\infty} J u^{j}: \sum_{j=1}^{\infty}\left\|u^{j}\right\|_{W^{1, n}}^{n}<\infty\right\}$ is of the first category in $\mathcal{H}^{1}\left(\mathbb{R}^{n}\right)$.

Corollary 1.3. The Jacobian operator does not map $W^{1, n}\left(\mathbb{R}^{n}, \mathbb{R}^{n}\right)$ onto $\mathcal{H}^{1}\left(\mathbb{R}^{n}\right)$ for any $n \geq 2$.

Most of the further examples of $\mathcal{H}^{1}$ regular compensated compactness quantities studied in 4] are bilinear operators or null Lagrangians. The former class is treated in Theorem 1.4 and 45 and the latter in 46 . We next describe the setting of Theorem 1.4 .

When $k \geq 0, n \geq 2, m, N \geq 1,1<p<\infty$, and $A: \mathcal{D}^{\prime}\left(\mathbb{R}^{n}, \mathbb{R}^{m}\right) \rightarrow \mathcal{D}^{\prime}\left(\mathbb{R}^{n}, \mathbb{R}^{N}\right)$ is a linear constant-coefficient, homogeneous partial differential operator (so that all the components of $A$ are of the form $\sum_{i=1}^{m} \sum_{|\alpha|=q} c_{\alpha, i} \partial^{\alpha} v_{i}$ with the same $q \in \mathbb{N}$ ), we denote

$$
\begin{aligned}
& W_{A}^{k, p}\left(\mathbb{R}^{n}, \mathbb{R}^{m}\right):=\quad\left\{v \in W^{k, p}\left(\mathbb{R}^{n}, \mathbb{R}^{m}\right): A v=0\right\}, \\
& C_{c, A}^{\infty}\left(\mathbb{R}^{n}, \mathbb{R}^{m}\right) \quad:=\quad\left\{v \in C_{c}^{\infty}\left(\mathbb{R}^{n}, \mathbb{R}^{m}\right): A v=0\right\} .
\end{aligned}
$$

Typical examples of $A$ in the Hardy space theory of compensated compactness are divergence, curl and the trivial operator $A=0$.

\footnotetext{
${ }^{1}$ Here and elsewhere, the notation $X \lesssim Y$ means $|X| \leq C Y$, where $C>0$ is a constant that depends on parameters that are rather clear from context, in this instance the dimension $n$. In order to smoothen the exposition we will not display dependence on dimensions of Euclidean spaces or exponents $k$ and $p$ when presenting estimates in terms of Sobolev norms $\|\cdot\|_{W^{k, p}}$ or seminorms $\left\|\nabla^{k} \cdot\right\|_{L^{p}}$, but such dependence is always tacitly assumed. The notation $X \lesssim_{B} Y$ is used when we wish to emphasize the dependence of the implicit constant $C$ on a parameter $B$.
} 
Various compensated compactness quantities are bilinear partial differential operators $\mathscr{B}: W_{A_{1}}^{k_{1}, p_{1}}\left(\mathbb{R}^{n}, \mathbb{R}^{m_{1}}\right) \times W_{A_{2}}^{k_{2}, p_{2}}\left(\mathbb{R}^{n}, \mathbb{R}^{m_{2}}\right) \rightarrow L^{1}\left(\mathbb{R}^{n}\right)$ of the form

$$
\mathscr{B}(u, v):=\sum_{i=1}^{m_{1}} \sum_{j=1}^{m_{2}} \sum_{|\alpha| \leq k_{1},|\beta| \leq k_{2}} c_{i, j, \alpha, \beta} \partial^{\alpha} u_{i} \partial^{\beta} v_{j}
$$

where

$$
\left.k_{1}, k_{2} \geq 0, n \geq 2, m_{1}, m_{2}, N \geq 1, p_{1}, p_{2} \in\right] 1, \infty\left[\text { with } \frac{1}{p_{1}}+\frac{1}{p_{2}}=1\right.
$$

(see $\$ 5.2$ for several examples).

Theorem 1.4. Suppose $\mathscr{B}: W_{A_{1}}^{k_{1}, p_{1}}\left(\mathbb{R}^{n}, \mathbb{R}^{m_{1}}\right) \times W_{A_{2}}^{k_{2}, p_{2}}\left(\mathbb{R}^{n}, \mathbb{R}^{m_{2}}\right) \rightarrow L^{1}\left(\mathbb{R}^{n}\right)$ is of the form (1.2), where the exponents satisfy (1.3) and $A_{1} \in\{0$, div, curl $\}$. The following conditions are equivalent.

(i) For every $(u, v) \in W_{A_{1}}^{k_{1}, p_{1}}\left(\mathbb{R}^{n}, \mathbb{R}^{m_{1}}\right) \times W_{A_{2}}^{k_{2}, p_{2}}\left(\mathbb{R}^{n}, \mathbb{R}^{m_{2}}\right)$,

$$
\int_{\mathbb{R}^{n}} \mathscr{B}(u, v)=0 .
$$

(ii) For every $(u, v) \in W_{A_{1}}^{k_{1}, p_{1}}\left(\mathbb{R}^{n}, \mathbb{R}^{m_{1}}\right) \times W_{A_{2}}^{k_{2}, p_{2}}\left(\mathbb{R}^{n}, \mathbb{R}^{m_{2}}\right)$,

$$
\|\mathscr{B}(u, v)\|_{\mathcal{H}^{1}} \lesssim\|u\|_{W^{k_{1}, p_{1}}}\|v\|_{W^{k_{2}, p_{2}}}
$$

Whenever $\mathscr{B}$ satisfies conditions (i)-(ii) and $k_{1} \geq 1$, the set

$$
\left\{\sum_{l=1}^{\infty} \lambda^{l} \mathscr{B}\left(u^{l}, v^{l}\right): \sup _{l \in \mathbb{N}}\left\|u^{l}\right\|_{W^{k_{1}, p_{1}}}\left\|v^{l}\right\|_{W^{k_{2}, p_{2}}} \leq 1, \sum_{l=1}^{\infty}\left|\lambda^{l}\right|<\infty\right\}
$$

is of the first category in $\mathcal{H}^{1}\left(\mathbb{R}^{n}\right)$.

When $|\alpha|=k_{1}$ and $|\beta|=k_{2}$ in all the terms of $\mathscr{B}$, the estimate in condition (ii) can be strengthened to $\|\mathscr{B}(u, v)\|_{\mathcal{H}^{1}} \lesssim\left\|\nabla^{k_{1}} u\right\|_{L^{p_{1}}}\left\|\nabla^{k_{2}} v\right\|_{L^{p_{2}}}$ - this fact is recorded in Theorem 4.5. When $A_{1}=A_{2}=0$, we give in Proposition 4.4 another condition equivalent to (i) and (ii) that refers directly to the coefficients of $\mathscr{B}$ and is rather easy to test.

This article is organized as follows. In $\ 2$ we present the necessary background for the article, whereas $\$ 3$ is devoted to Theorem [1.2 In $\$ 4$ we prove Theorem 1.4 and its modification for homogeneous Sobolev spaces, and applications are presented in \$5. Section 6 deals with null Lagrangians and more general homogeneous polynomials of partial derivatives, and open problems related to Question 1.1 are discussed in 97

\section{BACKGROUND}

2.1. $\mathcal{H}^{1}$ regularity of Jacobians. Good general references on the function spaces $\mathcal{H}^{1}\left(\mathbb{R}^{n}\right)$ and $\operatorname{BMO}\left(\mathbb{R}^{n}\right)$ include 16 and 41 . We fix, for the rest of this article, a function $\eta \in C_{c}^{\infty}\left(\mathbb{R}^{n}\right)$ with integral $\int_{\mathbb{R}^{n}} \eta \neq 0$ and denote $\eta_{t}(x):=t^{-n} \eta(x / t)$ for all $x \in \mathbb{R}^{n}$ and $t>0$. The real-variable Hardy space is defined by

$$
\mathcal{H}^{1}\left(\mathbb{R}^{n}\right):=\left\{h \in \mathcal{S}^{\prime}\left(\mathbb{R}^{n}\right): \sup _{t>0}\left|h * \eta_{t}\right| \in L^{1}\left(\mathbb{R}^{n}\right)\right\}
$$

and endowed with the norm $\|h\|_{\mathcal{H}^{1}}:=\left\|\sup _{t>0}\left|h * \eta_{t}\right|\right\|_{L^{1}}$. As shown by C. Fefferman and E. Stein in [12, any two choices of $\eta$ give equivalent norms for $\mathcal{H}^{1}\left(\mathbb{R}^{n}\right)$. By 
a famous theorem of $\mathrm{C}$. Fefferman, $\mathrm{BMO}\left(\mathbb{R}^{n}\right)$ is the dual of $\mathcal{H}^{1}\left(\mathbb{R}^{n}\right)$. The space $\operatorname{VMO}\left(\mathbb{R}^{n}\right)$ can be defined as the closure of $C_{c}^{\infty}\left(\mathbb{R}^{n}\right)$ in $\operatorname{BMO}\left(\mathbb{R}^{n}\right)$, and it was shown by R. Coifman and G. Weiss in [6] that $\mathcal{H}^{1}\left(\mathbb{R}^{n}\right)=\operatorname{VMO}\left(\mathbb{R}^{n}\right)^{*}$.

S. Müller showed in 34 that if $u \in W_{l o c}^{1, n}\left(\mathbb{R}^{n}, \mathbb{R}^{n}\right)$ with $J u \geq 0$ a.e. in an open set $\Omega$, then $J u \log (2+J u) \in L_{l o c}^{1}(\Omega)$, and motivated chiefly by Müller's theorem, R. Coifman, P.-L. Lions, Y. Meyer, and S. Semmes proved the following result.

Theorem 2.1 ( [4]). If $u=\left(u_{1}, \ldots, u_{n}\right) \in \dot{W}^{1, n}\left(\mathbb{R}^{n}, \mathbb{R}^{n}\right)$, then Ju belongs to $\mathcal{H}^{1}\left(\mathbb{R}^{n}\right)$ and the estimate $\|J u\|_{\mathcal{H}^{1}} \lesssim \prod_{j=1}^{n}\left\|\nabla u_{j}\right\|_{L^{n}}$ holds.

T. Iwaniec and J. Onninen subsequently weakened the hypotheses of Theorem 2.1 to $u \in W_{l o c}^{1, n-1}\left(\mathbb{R}^{n}, \mathbb{R}^{n}\right)$ and $|\operatorname{cof}(\mathrm{D} u)| \in L^{n /(n-1)}\left(\mathbb{R}^{n}\right)$ (and strengthened the conclusion to $\left.\|J u\|_{\mathcal{H}^{1}} \lesssim\|\operatorname{cof}(\mathrm{D} u)\|_{L^{n /(n-1)}}^{n /(n-1)}\right)$ in [23], answering a question raised by S. Müller, T. Qi and B.S. Yan in [33. For a unified way of proving Theorem 2.1 and $\mathcal{H}^{1}$ regularity results for numerous other quantities, involving e.g. fractional Laplacians, see [27. We also restate, for further reference, the Jacobian decomposition theorem mentioned in the introduction.

Theorem 2.2 ( [4]). For every $h \in \mathcal{H}^{1}\left(\mathbb{R}^{n}\right)$ there exist constans $\lambda^{j} \in \mathbb{R}$ and mappings $u^{j} \in W^{1, n}\left(\mathbb{R}^{n}, \mathbb{R}^{n}\right)$ with $\left\|\mathrm{D} u^{j}\right\|_{L^{n}} \leq 1$ such that

$$
h=\sum_{j=1}^{\infty} \lambda^{j} J u^{j} \quad \text { and } \quad \sum_{j=1}^{\infty}\left|\lambda^{j}\right| \lesssim\|h\|_{\mathcal{H}^{1}} .
$$

It would be impossible to cite here all the applications of Theorem 2.1 its variants, and the $\mathcal{H}^{1}$ regularity of other compensated compactness quantities, but we mention some examples. The higher regularity of Jacobians was first observed by H. Wente in his study of surfaces of constant mean curvature (see [45]), and Theorem 2.1 implies an improvement of an elliptic regularity result from [45] (see 4, p. 283] or [17, Theorem 3.4.1]). Other areas of application include, among others, weakly harmonic maps between manifolds ( [14, [17), geometric analysis in $\mathbb{R}^{n}$ ( [1], 22]), and mathematical fluid dynamics ( [30]) - the books cited above also contain lots of further references.

2.2. Sobolev spaces. We recall some properties of Sobolev spaces used in this article. For an introduction to homogeneous Sobolev spaces we refer to [26] and 40. When $n, m, k \in \mathbb{N}$ and $1<p<\infty$, we equip the homogeneous Sobolev space $\dot{W}^{k, p}\left(\mathbb{R}^{n}, \mathbb{R}^{m}\right):=\left\{u \in L_{\text {loc }}^{1}\left(\mathbb{R}^{n}, \mathbb{R}^{m}\right): \nabla^{k} u \in L^{p}\left(\mathbb{R}^{n}, \mathbb{R}^{k n m}\right)\right\}$ with the seminorm

$$
\|u\|_{\dot{W}^{k, p}}:=\left\|\nabla^{k} u\right\|_{L^{p}}:=\left(\sum_{i=1}^{m} \sum_{|\alpha|=k} \int_{\mathbb{R}^{n}}\left|\partial^{\alpha} u_{i}\right|^{p}\right)^{\frac{1}{p}},
$$

and $\dot{W}^{k, p}\left(\mathbb{R}^{n}, \mathbb{R}^{m}\right)$ is complete in $\|\cdot\|_{\dot{W}^{k, p}}$ (see [40, p. 188]). In order to define, as in (1.1),

$$
\dot{W}_{A}^{k, p}\left(\mathbb{R}^{n}, \mathbb{R}^{m}\right):=\left\{v \in \dot{W}^{k, p}\left(\mathbb{R}^{n}, \mathbb{R}^{m}\right): A v=0\right\}
$$

without ambiguities we do not identify polynomials of degree smaller than $k$. Smooth, compactly supported functions are dense in $\dot{W}^{k, p}\left(\mathbb{R}^{n}, \mathbb{R}^{m}\right.$ ) (see 40, pp. 194-195]). Furthermore, $\dot{W}^{k, p}\left(\mathbb{R}^{n}, \mathbb{R}^{m}\right) \subset W_{\text {loc }}^{k, p}\left(\mathbb{R}^{n}, \mathbb{R}^{m}\right)$ (see [18, Theorem 4.5.8]). Finally, we write $u^{j} \rightarrow u$ in $\dot{W}^{k, p}\left(\mathbb{R}^{n}, \mathbb{R}^{m}\right)$ when $\nabla^{k} u^{j} \rightarrow \nabla^{k} u$ in $L^{p}\left(\mathbb{R}^{n}, \mathbb{R}^{k n}\right)$.

We will repeatedly use a higher-order Poincaré inequality which is presented in the following lemma. A proof can be found e.g. at [39, pp. 2960-2961]. 
Lemma 2.3. Let $Q \subset \mathbb{R}^{n}$ be a cube and suppose $f \in W^{k, p}(Q)$, where $k \in \mathbb{N}$ and $1 \leq p<n$. Then there exists a polynomial $P_{Q}^{k-1} f$ of order at most $k-1$ such that

$$
f_{Q} \partial^{\alpha} P_{Q}^{k-1} f=f_{Q} \partial^{\alpha} f
$$

for every multi-index $\alpha \in(\mathbb{N} \cup\{0\})^{n}$ with $|\alpha| \leq k-1$. Furthermore,

$$
\begin{gathered}
\left(f_{Q}\left|f-P_{Q}^{k-1} f\right|^{\frac{n p}{n-k p}}\right)^{\frac{n-k p}{n p}} \lesssim l(Q)^{k}\left(f_{Q}\left|\nabla^{k} f\right|^{p}\right)^{\frac{1}{p}} \quad \text { if } k p<n, \\
\left(f_{Q}\left|f-P_{Q}^{k-1} f\right|^{q}\right)^{\frac{1}{q}} \lesssim l(Q)^{k}\left(f_{Q}\left|\nabla^{k} f\right|^{p}\right)^{\frac{1}{p}} \quad \text { if } k p \geq n \text { and } q<\infty,
\end{gathered}
$$

where the implicit constants do not depend on $Q$.

In 39, M. Prats and X. Tolsa state a modified version of inequalities (2.1) and (2.2), namely $\left\|f-P_{Q}^{k-1} f\right\|_{L^{p}(Q)} \lesssim l(Q)^{k}\left\|\nabla^{k} f\right\|_{L^{p}(Q)}$, but their proof also gives (2.1) and (2.2).

2.3. Complex analytical tools. We recall some tools of planar harmonic analysis and refer to [1] for the details, see also [29]. The homogeneous Sobolev space $\dot{W}^{1,2}(\mathbb{C}, \mathbb{C})$ is, up to identification of constant functions to zero, isomorphic to $L^{2}(\mathbb{C}, \mathbb{C})$, and an isomorphism is given by either of the Wirtinger derivatives

$$
u_{z}:=\frac{1}{2}\left(\frac{\partial}{\partial x}-i \frac{\partial}{\partial_{y}}\right)\left(u_{1}+i u_{2}\right) \quad \text { and } \quad u_{\bar{z}}:=\frac{1}{2}\left(\frac{\partial}{\partial x}+i \frac{\partial}{\partial y}\right)\left(u_{1}+i u_{2}\right) .
$$

The Beurling transform $\mathcal{S}: L^{2}(\mathbb{C}, \mathbb{C}) \rightarrow L^{2}(\mathbb{C}, \mathbb{C})$ is given by the Cauchy principal value integral

$$
\mathcal{S} f(z):=-\frac{1}{\pi} \lim _{\epsilon \searrow 0} \int_{\mathbb{C} \backslash B(0, \epsilon)} \frac{f(w)}{(z-w)^{2}} d w
$$

which converges a.e. when $f \in L^{2}(\mathbb{C}, \mathbb{C})$. The Beurling transform is an isometry in $L^{2}(\mathbb{C}, \mathbb{C})$, and $\mathcal{S}\left(u_{\bar{z}}\right)=u_{z}$ for all $u \in \dot{W}^{1,2}(\mathbb{C}, \mathbb{C})$. Thus, when $u \in \dot{W}^{1,2}(\mathbb{C}, \mathbb{C})$ and $f:=u_{\bar{z}} \in L^{2}(\mathbb{C}, \mathbb{C})$, we may write

$$
J u=\left|u_{z}\right|^{2}-\left|u_{\bar{z}}\right|^{2}=|\mathcal{S} f|^{2}-|f|^{2} .
$$

When $b \in \operatorname{BMO}(\mathbb{C})$, the complex linear operator $K_{b}: L^{2}(\mathbb{C}, \mathbb{C}) \rightarrow L^{2}(\mathbb{C}, \mathbb{C})$,

$$
K_{b} f:=\overline{(\mathcal{S} b-b \mathcal{S}) \overline{\mathcal{S f}}}
$$

defined and studied in [29], is self-adjoint. It allows us to rewrite a formula from [1. p. 547] in the form

$$
\int_{\mathbb{C}} b\left(|\mathcal{S} f|^{2}-|f|^{2}\right)=\int_{\mathbb{C}} f \overline{K_{b} f}
$$

By combining (2.4) and (2.5), the dual pairing of $b$ and $J u$ is expressed as an $L^{2}$ inner product. Furthermore, since $K_{b}$ is self-adjoint, its operator norm and numerical radius coincide, which implies the following characterization:

$$
\|b\|_{\mathrm{BMO}_{\mathcal{S}}}:=\sup _{\int_{\mathbb{C}}\left|u_{\bar{z}}\right|^{2}=1} \int_{\mathbb{C}} b J u=\sup _{\|f\|_{L^{2}}=1} \int_{\mathbb{C}} f \overline{K_{b} f}=\left\|K_{b}\right\|_{L^{2} \rightarrow L^{2}} .
$$


The norm $\|\cdot\|_{\mathrm{BMO}_{\mathcal{S}}}$ appeared, in a different guise, already in 4] where it was shown to be equivalent to $\|\cdot\|_{\mathrm{BMO}}$.

When $1<p<\infty$, the results of this subsection have natural analogues for $L^{p}(\mathbb{C}, \mathbb{C})$. In particular, (2.5) holds for every $b \in L^{p^{\prime}}(\mathbb{C})$ and $f \in L^{2 p}(\mathbb{C}, \mathbb{C})$.

2.4. Null Lagrangians. Jacobian, Hessian and minors of the Jacobian matrix belong to the class of null Lagrangians. In this article we confine ourselves to the null Lagrangians most relevant for compensated compactness theory, those of the form $\mathscr{L}\left(\nabla^{k} u\right)$. For further information on null Lagrangians we refer to [2], 21], [37, and 38.

Here and throughout the article we have striven, whenever possible, to use upper indices to enumerate elements of finite and infinite sequences and lower indices to enumerate components of multi-indices and mappings. We follow 2 but try to keep the notation consistent with the other parts of this article. In the rest of this subsection, $n, m, k \in \mathbb{N}$.

Definition 2.4. We denote by $X(n, m, k)$ the $m \times\left(\begin{array}{c}n+k-1 \\ k\end{array}\right)$-dimensional space of real matrices $V=\left[V_{i, \alpha}\right]$, where $1 \leq i \leq m$ and $\alpha \in(\mathbb{N} \cup\{0\})^{n}$ with $|\alpha|=k$.

The ordering of the components of elements of $X(n, m, k)$ is irrelevant for the arguments used in this article. When $u \in W^{k, r}\left(\mathbb{R}^{n}, \mathbb{R}^{m}\right)$, where $\left.r \in\right] 1, \infty[$, we denote $\nabla^{k} u:=\left[\partial^{\alpha} u_{i}\right]: \mathbb{R}^{n} \rightarrow X(n, m, k)$.

Definition 2.5. A continuous function $\mathscr{L}: X(n, m, k) \rightarrow \mathbb{R}$ is called a null Lagrangian if

$$
\int_{\Omega} \mathscr{L}\left(\nabla^{k}(u+\varphi)\right)=\int_{\Omega} \mathscr{L}\left(\nabla^{k} u\right)
$$

for every bounded open set $\Omega \subset \mathbb{R}^{n}$ and every $u \in C^{k}(\bar{\Omega})$ and $\varphi \in C_{0}^{\infty}(\Omega)$.

Null Lagrangians are also called quasiaffine functions in the literature. The following result contains part of [2, Theorem 3.4]; condition (iii) is presented in a slightly different form in [2].

Theorem 2.6 ([2]). Let $\mathscr{L}: X(n, m, k) \rightarrow \mathbb{R}$ be continuous. The following conditions are equivalent:

(i) $\mathscr{L}$ is a null Lagrangian.

(ii) $\int_{\Omega} \mathscr{L}\left(c+\nabla^{k} \varphi\right)=\int_{\Omega} \mathscr{L}(c)$ for every bounded open set $\Omega \subset \mathbb{R}^{n}$ and all $\varphi \in C_{c}^{\infty}\left(\Omega, \mathbb{R}^{m}\right)$ and $c \in X(n, m, k)$.

(iii) $\mathscr{L}$ is a polynomial (of degree $r$, say) and furthermore, whenever $u^{j} \rightarrow u$ in $\dot{W}^{k, r}\left(\mathbb{R}^{n}, \mathbb{R}^{m}\right)$, we have $\mathscr{L}\left(\nabla^{k} u^{j}\right) \rightarrow \mathscr{L}\left(\nabla^{k} u\right)$ in $\mathcal{D}^{\prime}\left(\mathbb{R}^{n}\right)$.

Another theorem from [2] says that $\mathscr{L}$ is a null Lagrangian if and only if $\mathscr{L}$ is an affine combination of minors of $\nabla^{k} u$. This characterization implies the following result which is also a special case of Theorem 6.2.

Proposition 2.7. Suppose that a null Lagrangian $\mathscr{L}: X(n, m, k) \rightarrow \mathbb{R}$ is a polynomial of degree $r \geq 2$. The inequality $\left\|\mathscr{L}\left(\nabla^{k} u\right)\right\|_{\mathcal{H}^{1}} \lesssim\left\|\nabla^{k} u\right\|_{L^{r}}^{r}$ holds for all $u \in \dot{W}^{k, r}\left(\mathbb{R}^{n}, \mathbb{R}^{m}\right)$ if and only if $\mathscr{L}$ is $r$-homogeneous.

In $\$ 6$ we give an example of an homogeneous polynomial $\mathscr{L}: X(3,2,2) \rightarrow \mathbb{R}$ that satisfies the estimate $\left\|\mathscr{L}\left(\nabla^{2} u\right)\right\|_{\mathcal{H}^{1}} \lesssim\left\|\nabla^{2} u\right\|_{L^{3}}^{3}$ for all $u \in \dot{W}^{2,3}\left(\mathbb{R}^{3}, \mathbb{R}^{2}\right)$ but fails to be be a null Lagrangian. 
2.5. Potentials for divergence and curl. It is the purpose of this subsection to find, given $k \in \mathbb{N} \cup\{0\}$ and $1<p<\infty$, a first-order homogeneous partial differential operator $B: \dot{W}^{k+1, p}\left(\mathbb{R}^{n}, \mathbb{R}^{n(n-1) / 2}\right) \rightarrow \dot{W}_{\text {div }}^{k, p}\left(\mathbb{R}^{n}, \mathbb{R}^{n}\right)$ such that every $u \in \dot{W}_{\text {div }}^{k, p}\left(\mathbb{R}^{n}, \mathbb{R}^{n}\right)$ can be written as $u=B \Phi$, where $\left\|\nabla^{k+1} \Phi\right\|_{L^{p}} \approx\left\|\nabla^{k} u\right\|_{L^{p}}$. The result is in effect a Poincaré lemma with suitable norm control: given $u \in$ $\dot{W}_{\text {div }}^{k, p}\left(\mathbb{R}^{n}, \mathbb{R}^{n}\right)$ the idea is to define the closed $n-1$ form $\alpha:=*\left(\sum_{j=1}^{n} u_{j} d x_{j}\right)$ and then find a suitable $n-2$ form $\beta$ with $d \beta=\alpha$. We will however avoid the use of differential forms in the exposition. We also present analogous results for curl.

We denote the vector space of strictly upper triangular $n \times n$ matrices by $\mathbb{R}_{\Delta}^{n \times n}$.

Definition 2.8. We define $C: \mathcal{D}^{\prime}\left(\mathbb{R}^{n}, \mathbb{R}^{n}\right) \rightarrow \mathcal{D}^{\prime}\left(\mathbb{R}^{n}, \mathbb{R}_{\Delta}^{n \times n}\right)$ by

$$
C(T):=\left[(-1)^{i+j} \chi_{i<j}(i, j)\left(\partial_{j} T_{i}-\partial_{i} T_{j}\right)\right]_{i, j=1}^{n}
$$

and $B: \mathcal{D}^{\prime}\left(\mathbb{R}^{n}, \mathbb{R}_{\Delta}^{n \times n}\right) \rightarrow \mathcal{D}_{\text {div }}^{\prime}\left(\mathbb{R}^{n}, \mathbb{R}^{n}\right)$ by

$$
B(\Phi):=\prod_{j=1}^{n}\left(\sum_{i<j}(-1)^{i+j} \partial_{i} \Phi_{i j}+\sum_{i>j}(-1)^{i+j+1} \partial_{i} \Phi_{j i}\right) .
$$

A straightforward computation gives the identity $-\Delta T=B \circ C(T)$ for all divergence-free $T \in \mathcal{D}^{\prime}\left(\mathbb{R}^{n}, \mathbb{R}^{n}\right)$. In order to use the identity we prove the following lemma.

Lemma 2.9. Let $k \in \mathbb{N} \cup\{0\}$ and $1<q<n$. If $u \in L_{\text {div }}^{q}\left(\mathbb{R}^{n}, \mathbb{R}^{n}\right)$, then the Poisson equation $-\Delta T=u$ has a solution $T \in \dot{W}_{\text {div }}^{2, q}\left(\mathbb{R}^{n}, \mathbb{R}^{n}\right) \cap \dot{W}^{1, n q /(n-q)}\left(\mathbb{R}^{n}, \mathbb{R}^{n}\right)$ that is unique up to an additive constant.

Proof. We first prove the existence statement. Let $u \in L_{\text {div }}^{q}\left(\mathbb{R}^{n}, \mathbb{R}^{n}\right)$ and choose $\varphi^{k} \in C_{c, \text { div }}^{\infty}\left(\mathbb{R}^{n}, \mathbb{R}^{n}\right)$ such that $\left\|\varphi^{k}-u\right\|_{L^{q}} \rightarrow 0$ as $k \rightarrow \infty$ (see e.g. [13, Lemma III.2.1]). Now the sequence $\left(-\Delta\left[\varphi^{k} * G\right]\right)_{k=1}^{\infty}=\left(\varphi^{k}\right)_{k=1}^{\infty}$ is Cauchy in $L^{q}\left(\mathbb{R}^{n}, \mathbb{R}^{n}\right)$, where $G=-(2 \pi)^{-1} \log |\cdot|$ when $n=2$ and $G=\left(n(n-2) \omega_{n}\right)^{-1}|\cdot|^{2-n}$ when $n \geq 3$. Thus $\left(\nabla^{2}\left[\varphi^{k} * G\right]\right)_{k=1}^{\infty}$ is Cauchy in $L^{q}\left(\mathbb{R}^{n}, \mathbb{R}^{n^{3}}\right)$.

Now $\mathrm{D}\left[\varphi^{k} * G\right] \in L^{n q /(n-q)}\left(\mathbb{R}^{n}, \mathbb{R}^{n \times n}\right)$ for every $k \in \mathbb{N}$. By the SobolevGagliardo-Nirenberg theorem, $\left(\mathrm{D}\left[\varphi^{k} * G\right]\right)_{k=1}^{\infty}$ is Cauchy in $L^{n q /(n-q)}\left(\mathbb{R}^{n}, \mathbb{R}^{n \times n}\right)$. Hence, for every $i \in\{1, \ldots, n\}$ there exists $f_{i} \in \dot{W}^{1, q}\left(\mathbb{R}^{n}, \mathbb{R}^{n}\right) \cap L^{n q /(n-q)}\left(\mathbb{R}^{n}, \mathbb{R}^{n}\right)$ such that $\left\|\nabla^{2}\left[\varphi_{i}^{k} * G\right]-\mathrm{D} f_{i}\right\|_{L^{q}} \rightarrow 0$ and $\left\|\nabla\left[\varphi_{i}^{k} * G\right]-f_{i}\right\|_{L^{n q /(n-q)}} \rightarrow 0$. Now $f_{i}$ is curl-free and thus $f_{i}=\nabla T_{i}$, where $T_{i} \in \dot{W}^{2, q}\left(\mathbb{R}^{n}\right) \cap \dot{W}^{1, n q /(n-q)}\left(\mathbb{R}^{n}\right)$. Consequently, $T:=\left(T_{1}, \ldots, T_{n}\right)$ satisfies $\operatorname{div} T=\lim _{k \rightarrow \infty} \operatorname{div} \varphi^{k} * G=0$ and $-\Delta T=u$.

The uniqueness statement is proved as follows. Suppose $\tilde{T} \in \dot{W}_{\operatorname{div}}^{2, q}\left(\mathbb{R}^{n}, \mathbb{R}^{n}\right) \cap$ $\dot{W}^{1, n q /(n-q)}\left(\mathbb{R}^{n}, \mathbb{R}^{n}\right)$ satisfies $\Delta \tilde{T}=0$. Then $\mathrm{D} \tilde{T} \in L^{n q /(n-q)}\left(\mathbb{R}^{n}, \mathbb{R}^{n \times n}\right)$ is harmonic and therefore vanishes, and so $\tilde{T}$ is constant.

Before producing the potential for divergence recall that when $1<p<\infty$ and $R=\left(R_{1}, \ldots, R_{n}\right)$, the operator $I+R \otimes R: L^{p}\left(\mathbb{R}^{n}, \mathbb{R}^{n}\right) \rightarrow L^{p}\left(\mathbb{R}^{n}, \mathbb{R}^{n}\right)$ is a projection onto divergence-free vector fields.

Proposition 2.10. Let $k \in \mathbb{N} \cup\{0\}$ and $1<p<\infty$. The linear operator $B: \dot{W}^{k+1, p}\left(\mathbb{R}^{n}, \mathbb{R}_{\Delta}^{n \times n}\right) \rightarrow \dot{W}_{\text {div }}^{k, p}\left(\mathbb{R}^{n}, \mathbb{R}^{n}\right)$ has a linear right inverse $u \mapsto \Phi$ such that $\left\|\nabla^{k+1} \Phi\right\|_{L^{p}} \approx\left\|\nabla^{k} u\right\|_{L^{p}}$ for all $u \in \dot{W}_{\text {div }}^{k, p}\left(\mathbb{R}^{n}, \mathbb{R}^{n}\right)$. Furthermore, $\nabla^{k} u^{j} \rightarrow \nabla^{k} u$ in $L^{p}\left(\mathbb{R}^{n}, \mathbb{R}^{k n}\right)$ if and only if $\nabla^{k+1} \Phi^{j} \rightarrow \nabla^{k+1} \Phi$ in $L^{p}\left(\mathbb{R}^{n}, \mathbb{R}^{(k+1) n(n-1) / 2}\right)$. 
Proof. It suffices to show that the linear right inverse exists in a dense subset of $\dot{W}_{\text {div }}^{k, p}\left(\mathbb{R}^{n}, \mathbb{R}^{n}\right)$. Seeking a suitable dense set, let $u \in \dot{W}_{\text {div }}^{k, p}\left(\mathbb{R}^{n}, \mathbb{R}^{n}\right)$ and choose $u^{j} \in C_{c}^{\infty}\left(\mathbb{R}^{n}, \mathbb{R}^{n}\right)$ such that $\left\|\nabla^{k}\left(u^{j}-u\right)\right\|_{L^{p}} \rightarrow 0$. When $\alpha \in(\mathbb{N} \cup\{0\})^{n}$ with $|\alpha|=k$, note that $\partial^{\alpha} u:=\left(\partial^{\alpha} u_{1}, \ldots, \partial^{\alpha} u_{n}\right) \in L_{\text {div }}^{p}\left(\mathbb{R}^{n}, \mathbb{R}^{n}\right)$ and thus $(I+R \otimes R) \partial^{\alpha} u=\partial^{\alpha} u$. Now

$$
\partial^{\alpha}(I+R \otimes R) u^{j}=(I+R \otimes R) \partial^{\alpha} u^{j} \rightarrow(I+R \otimes R) \partial^{\alpha} u=\partial^{\alpha} u
$$

in $L^{p}\left(\mathbb{R}^{n}, \mathbb{R}^{n}\right)$. We conclude that $\left\{(I+R \otimes R) \varphi: \varphi \in C_{c}^{\infty}\left(\mathbb{R}^{n}, \mathbb{R}^{n}\right)\right\}$ is dense in $\dot{W}_{\text {div }}^{k, p}\left(\mathbb{R}^{n}, \mathbb{R}^{n}\right)$.

Fix now $q \in] 1, n\left[\right.$ and let $\varphi \in C_{c}^{\infty}\left(\mathbb{R}^{n}, \mathbb{R}^{n}\right)$. Use Lemma 2.9 to choose a solution $T \in \dot{W}_{\text {div }}^{2, q}\left(\mathbb{R}^{n}, \mathbb{R}^{n}\right) \cap \dot{W}^{1, n q /(n-q)}\left(\mathbb{R}^{n}, \mathbb{R}^{n}\right)$ of the Poisson equation $-\Delta T=(I+$ $R \otimes R) \varphi$. Now $\Phi:=C(T)$ is well-defined and $B \Phi=(I+R \otimes R) \varphi$. Moreover, $(I+R \otimes R) \varphi \mapsto \Phi$ is linear. Trivially $\left\|\nabla^{k+1} \Phi\right\|_{L^{p}} \lesssim\left\|\nabla^{k+2} T\right\|_{L^{p}}$, whereas for every $\alpha \in(\mathbb{N} \cup\{0\})^{n}$ with $|\alpha|=k+2$ we write $\alpha=(\alpha-\gamma)+\gamma$ with $|\gamma|=2$ and obtain

$$
\left\|\partial^{\alpha} T\right\|_{L^{p}}=\left\|R^{\gamma} \partial^{\alpha-\gamma}(I+R \otimes R) \varphi\right\|_{L^{p}} \lesssim\left\|\nabla^{k}(I+R \otimes R) \varphi\right\|_{L^{p}},
$$

where $R^{\gamma}$ is the second-order Riesz transform corresponding to $\gamma$. The converse estimate $\left\|\nabla^{k}(I+R \otimes R) \varphi\right\|_{L^{p}}=\left\|\nabla^{k} B \Phi\right\|_{L^{p}} \lesssim\left\|\nabla^{k+1} \Phi\right\|_{L^{p}}$ is obvious.

We next show the equivalence of weak convergences. Clearly $\nabla^{k+1} \Phi^{j} \rightarrow \nabla^{k+1} \Phi$ implies $\nabla^{k} u^{j} \rightarrow \nabla^{k} u$. For the other direction suppose $\nabla^{k} u^{j} \rightarrow \nabla^{k} u$ and let $\epsilon>0$. Choose $u_{\epsilon} \in C_{c, \text { div }}^{\infty}\left(\mathbb{R}^{n}, \mathbb{R}^{n}\right)$ such that $\left\|\nabla^{k} u_{\epsilon}-\nabla^{k} u\right\|_{L^{p}}<\epsilon$. For every $j \in \mathbb{N}$ select $u_{\epsilon}^{j} \approx u_{\epsilon}+u^{j}-u$ in $C_{c, \text { div }}^{\infty}\left(\mathbb{R}^{n}, \mathbb{R}^{n}\right)$ such that $\left\|\nabla^{k} u_{\epsilon}^{j}-\nabla^{k} u^{j}\right\|_{L^{p}}<2 \epsilon$ and $\nabla^{k} u_{\epsilon}^{j} \rightarrow \nabla^{k} u_{\epsilon}$. It now suffices to note that $\nabla^{k} u_{\epsilon}^{j} \rightarrow \nabla^{k} u_{\epsilon}$ implies, as above, $\partial^{\alpha} T_{\epsilon}^{j}=$ $R^{\gamma} \partial^{\alpha-\gamma} u_{\epsilon}^{j} \rightarrow R^{\gamma} \partial^{\alpha-\gamma} u_{\epsilon}=\partial^{\alpha} T_{\epsilon}$ for every $\alpha \in(\mathbb{N} \cup\{0\})^{n}$ with $|\alpha|=k+2$, which in turn implies that $\nabla^{k+1} \Phi_{\epsilon}^{j} \rightarrow \nabla^{k+1} \Phi_{\epsilon}$. We conclude that $\nabla^{k+1} \Phi^{j} \rightarrow \nabla^{k+1} \Phi$.

In $\$ 4.2$ we need the following variant of Proposition 2.10

Proposition 2.11. Let $k \in \mathbb{N} \cup\{0\}$ and $1<p<\infty$. The linear operator $B: \cap_{l=1}^{k+1} \dot{W}^{l, p}\left(\mathbb{R}^{n}, \mathbb{R}_{\Delta}^{n \times n}\right) \rightarrow W_{\mathrm{div}}^{k, p}\left(\mathbb{R}^{n}, \mathbb{R}^{n}\right)$ has a linear right inverse $u \mapsto \Phi$ such that $\sum_{l=1}^{k+1}\left\|\nabla^{l} \Phi\right\|_{L^{p}} \approx\|u\|_{W^{k, p}}$ for all $u \in W_{\mathrm{div}}^{k, p}\left(\mathbb{R}^{n}, \mathbb{R}^{n}\right)$.

Proof. When $u \in W_{\text {div }}^{k, p}\left(\mathbb{R}^{n}, \mathbb{R}^{n}\right)$, again we choose mappings $u^{j} \in C_{c}^{\infty}\left(\mathbb{R}^{n}, \mathbb{R}^{n}\right)$ such that $\left\|u^{j}-u\right\|_{W^{k, p}} \rightarrow 0$, solve the Poisson equation $-\Delta T^{j}=(I+R \otimes R) u^{j}$ and set $\Phi^{j}:=C\left(T^{j}\right)$. Now $\left(\Phi^{j}\right)_{j=1}^{\infty}$ is a Cauchy sequence in $\dot{W}^{l, p}\left(\mathbb{R}^{n}, \mathbb{R}_{\Delta}^{n \times n}\right)$ for every $l \in\{1, \ldots, k+1\}$ and therefore $\left(\mathrm{D} \Phi^{j}\right)_{j=1}^{\infty}$ has a limit $w$ in $W^{k, p}\left(\mathbb{R}^{n}, \mathbb{R}^{n^{2}(n-1) / 2}\right)$. On the other hand, there exists $\Phi \in \dot{W}^{1, p}\left(\mathbb{R}^{n}, \mathbb{R}_{\Delta}^{n \times n}\right)$ such that $\left\|\mathrm{D} \Phi^{j}-\mathrm{D} \Phi\right\|_{L^{p}} \rightarrow 0$ and so $\mathrm{D} \Phi=w \in W^{k, p}\left(\mathbb{R}^{n}, \mathbb{R}^{n^{2}(n-1) / 2}\right)$. As in the proof above, $\sum_{l=1}^{k+1}\left\|\nabla^{l} \Phi\right\|_{L^{p}} \approx$ $\|\mathrm{D} \Phi\|_{W^{k, p}} \approx\|u\|_{W^{k, p}}$.

Analogous results of course hold for $W_{\text {curl }}^{k, p}\left(\mathbb{R}^{n}, \mathbb{R}^{n}\right)$ and $\dot{W}_{\text {curl }}^{k, p}\left(\mathbb{R}^{n}, \mathbb{R}^{n}\right)$.

Proposition 2.12. Let $k \in \mathbb{N} \cup\{0\}$ and $1<p<\infty$. If $u \in \dot{W}_{\text {curl }}^{k, p}\left(\mathbb{R}^{n}, \mathbb{R}^{n}\right)$, then there exists $\Psi \in \dot{W}^{k+1, p}\left(\mathbb{R}^{n}\right)$ such that $\nabla \Psi=u$ and $\left\|\nabla^{k+1} \Psi\right\|_{L^{p}} \approx\left\|\nabla^{k} u\right\|_{L^{p}}$. If, furthermore, $u \in W_{\text {curl }}^{k, p}\left(\mathbb{R}^{n}, \mathbb{R}^{n}\right)$, then $\sum_{l=1}^{k+1}\left\|\nabla^{l} \Psi\right\|_{L^{p}} \approx\|u\|_{W^{k, p}}$.

We also record the following fact.

Proposition 2.13. Suppose $k \in \mathbb{N} \cup\{0\}$ and $1<p<\infty$. Then $C_{c, \mathrm{div}}^{\infty}\left(\mathbb{R}^{n}, \mathbb{R}^{n}\right)$ is dense in $\dot{W}_{\text {div }}^{k, p}\left(\mathbb{R}^{n}, \mathbb{R}^{n}\right)$ and $C_{c, \text { curl }}^{\infty}\left(\mathbb{R}^{n}, \mathbb{R}^{n}\right)$ is dense in $\dot{W}_{\text {curl }}^{k, p}\left(\mathbb{R}^{n}, \mathbb{R}^{n}\right)$. 
Proof. Let $u \in \dot{W}_{\text {div }}^{k, p}\left(\mathbb{R}^{n}, \mathbb{R}^{n}\right)$ and choose $\Phi \in \dot{W}^{k+1, p}\left(\mathbb{R}^{n}, \mathbb{R}_{\Delta}^{n \times n}\right)$ with $B \Phi=$ $u$. Select $\Phi^{j} \in C_{c}^{\infty}\left(\mathbb{R}^{n}, \mathbb{R}_{\Delta}^{n \times n}\right)$ such that $\Phi^{j} \rightarrow \Phi$ in $\dot{W}^{k+1, p}\left(\mathbb{R}^{n}, \mathbb{R}_{\Delta}^{n \times n}\right)$. Then $C_{c, \text { div }}^{\infty}\left(\mathbb{R}^{n}, \mathbb{R}^{n}\right) \ni B\left(\Phi^{j}\right) \rightarrow B(\Phi)=u$ in $\dot{W}_{\text {div }}^{k, p}\left(\mathbb{R}^{n}, \mathbb{R}^{n}\right)$. A similar proof applies to $\dot{W}_{\text {curl }}^{k, p}\left(\mathbb{R}^{n}, \mathbb{R}^{n}\right)$.

\section{Proof of Theorem 1.2}

Theorem 1.2 is proved in this section, and we divide the proof into two parts. In 3.1 we present an extension of a functional analytical lemma from [4, and the proof is finished in $\$ 3.2$. In $\$ 3.3$ we show that, as a corollary of Theorem 1.2, we have very little control on the norms of solutions of the Jacobian equation.

3.1. A functional analytical lemma. In the proof of Theorem 2.2 Coifman, Lions, Meyer, and Semmes used functional analytical lemmas 4, Lemmas III.1III.2] which give equivalent conditions for the existence of atomic decompositions in Banach spaces. We add to their criteria a further characterization that can be used when an atomic decomposition does not exist.

When $X$ is a Banach space and $V \subset X$ is bounded, recall that the $s$-convex hull of $V$ is defined by

$$
\mathrm{s}(V):=\left\{\sum_{j=1}^{\infty} \lambda^{j} x^{j}: \lambda^{j} \geq 0 \text { and } x^{j} \in V \text { for all } j \in \mathbb{N}, \sum_{j=1}^{\infty} \lambda^{j}=1\right\} .
$$

It is easy to show that $\operatorname{co}(V) \subset \mathrm{s}(V) \subset \overline{\mathrm{co}}(V)$, and thus $\overline{\mathrm{s}(V)}=\overline{\mathrm{co}}(V)$.

Lemma 3.1. Suppose $V$ is a symmetric, bounded subset of a Banach space $X$. The following conditions are equivalent.

(i) $\|l\|_{X^{*}}$ and $\sup _{x \in V}\langle l, x\rangle$ are equivalent norms in $X^{*}$.

(ii) $\overline{\mathrm{co}}(V)$ contains a ball centered at the origin.

(iii) $\mathrm{s}(V)$ contains a ball centered at the origin.

(iv) The set $\left\{\sum_{j=1}^{\infty} \lambda^{j} x^{j}: x^{j} \in V \forall j \in \mathbb{N}, \sum_{j=1}^{\infty}\left|\lambda^{j}\right|<\infty\right\}$ is of the second category in $X$.

The equivalence of $(i)-(i i i)$ is proved at [4, pp. 262-264], and direction (iii) $\Rightarrow$ (iv) follows from the Baire category theorem. In this article we only use implication $(i v) \Rightarrow(i)$.

Proof of direction (iv) $\Rightarrow(i)$. Suppose (iv) holds. Since $V$ is symmetric,

$$
\left\{\sum_{j=1}^{\infty} \lambda^{j} x^{j}: x^{j} \in V \forall j \in \mathbb{N}, \sum_{j=1}^{\infty}\left|\lambda^{j}\right|<\infty\right\}=\bigcup_{k=1}^{\infty} k \mathrm{~s}(V) .
$$

By assumption, there exists $k \in \mathbb{N}$ such that $\overline{k \mathrm{~s}(V)}=k \overline{\operatorname{co}}(V)$ contains a ball $B\left(x_{0}, \delta\right)$, and so $B(0,2 \delta)=B\left(x_{0}, \delta\right)-B\left(x_{0}, \delta\right) \subset 2 k \overline{\operatorname{co}}(V)$. When $l \in X^{*}$, we use the previous set inclusion and the boundedness of $V$ to get, as in [4,

$$
\frac{\delta}{k}\|l\|_{X^{*}} \leq \sup _{x \in \overline{\operatorname{co}}(V)}\langle l, x\rangle=\sup _{x \in \overline{\operatorname{co}}(V)}|\langle l, x\rangle|=\sup _{x \in V}|\langle l, x\rangle|=\sup _{x \in V}\langle l, x\rangle \leq C\|l\|_{X^{*}}
$$




\subsection{Completion of the proof. We denote}

$$
V:=\left\{J u:\|u\|_{W^{1, n}} \leq 1\right\} \subset \mathcal{H}^{1}\left(\mathbb{R}^{n}\right)
$$

and set out to show that the norms $\|b\|_{\text {BMO }}$ and $\sup _{h \in V} \int_{\mathbb{R}^{n}} b h$ are not equivalent in $\operatorname{BMO}\left(\mathbb{R}^{n}\right)$. Theorem 1.2 then follows from Lemma 3.1. The basic phenomenon behind the proof is the incompatibility of the scaling properties of $W^{1, n}\left(\mathbb{R}^{n}, \mathbb{R}^{n}\right)$ and $\operatorname{BMO}\left(\mathbb{R}^{n}\right)=\mathcal{H}^{1}\left(\mathbb{R}^{n}\right)^{*}$.

When $\tau>0$, the change of variables $y=\tau^{-1} x$ yields the formulas

$$
\begin{gathered}
\|b(\tau \cdot)\|_{\mathrm{BMO}}=\|b\|_{\mathrm{BMO}}, \\
\int_{\mathbb{R}^{n}}|u(\tau \cdot)|^{n}+\sum_{i, j=1}^{n} \int_{\mathbb{R}^{n}}\left|\partial_{j}\left[u_{i}(\tau \cdot)\right]\right|^{n}=\tau^{-n} \int_{\mathbb{R}^{n}}|u|^{n}+\sum_{i, j=1}^{n} \int_{\mathbb{R}^{n}}\left|\partial_{j} u_{i}\right|^{n}, \\
\int_{\mathbb{R}^{n}} b(\tau \cdot) J[u(\tau \cdot)]=\int_{\mathbb{R}^{n}} b J u
\end{gathered}
$$

for all $b \in \operatorname{BMO}\left(\mathbb{R}^{n}\right)$ and $u \in W^{1, n}\left(\mathbb{R}^{n}, \mathbb{R}^{n}\right)$.

Proof of Theorem 1.2. Seeking contradiction, assume that

$$
\|b\|_{\mathrm{BMO}} \lesssim \sup _{\|u\|_{W^{1, n}} \leq 1} \int_{\mathbb{C}} b J u=\sup _{u \neq 0} \frac{\int_{\mathbb{C}} b J u}{\|u\|_{W^{1, n}}^{n}}
$$

for all $b \in \operatorname{BMO}\left(\mathbb{R}^{n}\right)$. Fix $b \in C_{c}^{\infty}\left(\mathbb{R}^{n}\right)$ with $\|b\|_{\mathrm{BMO}}=1$.

Let $\tau>0$. By (3.1)-(3.4), there exists $v=u(\tau \cdot) \in W^{1, n}\left(\mathbb{R}^{n}, \mathbb{R}^{n}\right) \backslash\{0\}$ such that

$$
\begin{aligned}
1 & =\|b(\tau \cdot)\|_{\mathrm{BMO}} \lesssim \frac{\int_{\mathbb{C}} b(\tau \cdot) J v}{\int_{\mathbb{R}^{n}}|v|^{n}+\sum_{i, j=1}^{n} \int_{\mathbb{R}^{n}}\left|\partial_{j} v_{i}\right|^{n}} \\
& =\frac{\int_{\mathbb{R}^{n}} b J u}{\tau^{-n} \int_{\mathbb{R}^{n}}|u|^{n}+\sum_{i, j=1}^{n} \int_{\mathbb{R}^{n}}\left|\partial_{j} u_{i}\right|^{n}} .
\end{aligned}
$$

In (3.5) the mapping $u$ depends on $\tau$. Our goal is to show that when $\tau>0$ is small enough, (3.5) leads to a contradiction.

Let $M>0$ and use integration by parts and Young's inequality to get

$$
\begin{aligned}
\int_{\mathbb{R}^{n}} b J u & =-\int_{\mathbb{R}^{n}} u_{1} J\left(b, u_{2}, \ldots, u_{n}\right) \\
& \leq \int_{\mathbb{R}^{n}}\left(\frac{1}{n}\left(M\left|u_{1}\right|\right)^{n}+\frac{n-1}{n}\left(\frac{\left|J\left(b, u_{2}, \ldots, u_{n}\right)\right|}{M}\right)^{\frac{n}{n-1}}\right) \\
& \leq \frac{1}{n} M^{n} \int_{\mathbb{R}^{n}}|u|^{n}+\frac{n-1}{n} \frac{\|\nabla b\|_{L^{\infty}}^{\frac{n}{n-1}}}{M^{\frac{n}{n-1}}} \int_{\mathbb{R}^{n}}|\mathrm{D} u|^{n},
\end{aligned}
$$

where we used the Hadamard inequality $\left|J\left(b, u_{2}, \ldots, u_{n}\right)\right| \leq|\nabla b| \prod_{j=2}^{n}\left|\nabla u_{j}\right|$. By combining the previous estimate with (3.5) we get

$$
\begin{aligned}
& \tau^{-n} \int_{\mathbb{R}^{n}}|u|^{n}+\sum_{i, j=1}^{n} \int_{\mathbb{R}^{n}}\left|\partial_{j} u_{i}\right|^{n} \\
\leq & C(n)\left(M^{n} \int_{\mathbb{R}^{n}}|u|^{n}+\frac{\|\nabla b\|_{L^{\frac{n}{n-1}}}^{\frac{n}{n-1}}}{M^{\frac{n}{n-1}}} \sum_{i, j=1}^{n} \int_{\mathbb{R}^{n}}\left|\partial_{j} u_{i}\right|^{n}\right) .
\end{aligned}
$$


When we choose $M$ large enough and then $\tau$ small enough (both depending on $b$ ), a mapping $u \in W^{1, n}\left(\mathbb{R}^{n}, \mathbb{R}^{n}\right) \backslash\{0\}$ satisfying (3.6) cannot exist, and so we have obtained a contradiction.

3.3. Failure of norm control. Despite Theorem 1.2 and Corollary 1.3 , the range of $J: W^{1, n}\left(\mathbb{R}^{n}, \mathbb{R}^{n}\right) \rightarrow \mathcal{H}^{1}\left(\mathbb{R}^{n}\right)$ is dense in $\mathcal{H}^{1}\left(\mathbb{R}^{n}\right)$. This follows from the remarkable work of G. Cupini, B. Dacorogna and O. Kneuss on the Jacobian equation in [7, Theorem 1] and more directly from [25, Theorem 1]. However, solutions of the Jacobian equation utterly lack norm control, as we show in this subsection.

A mapping $u \in W^{1, n}\left(\mathbb{R}^{n}, \mathbb{R}^{n}\right)$ is said to be a minimum norm solution if $\|u\|_{W^{1, n}}=$ $\min \left\{\|v\|_{W^{1, n}}: J v=J u\right\}$. If $h \in \mathcal{H}^{1}\left(\mathbb{R}^{n}\right)$ and the Jacobian equation $J u=h$ has a solution, then the equation has a minimum norm solution; this follows from the weak continuity of the Jacobian and the direct method of the calculus of variations.

Proposition 3.2. Let $C>0$. Then the set

$$
\left\{J u: u \text { is a minimum norm solution, }\|u\|_{W^{1, n}}^{n} \leq C\|J u\|_{\mathcal{H}^{1}}\right\}
$$

is nowhere dense in $\mathcal{H}^{1}\left(\mathbb{R}^{n}\right)$ and the set

$$
\left\{J u: u \text { is a minimum norm solution, }\|u\|_{W^{1, n}}^{n}>C\|J u\|_{\mathcal{H}^{1}}\right\}
$$

is dense in $\mathcal{H}^{1}\left(\mathbb{R}^{n}\right)$.

Proof. We use a proof by contradiction to deduce the first statement. Suppose \{Ju: $\left.\|u\|_{W^{1, n}}^{n} \leq C\|J u\|_{\mathcal{H}^{1}}\right\}$ contains a ball. Then, for some $M>0$, the bounded, symmetric set

$$
\left.V:=\overline{\left\{ \pm M J u:\|u\|_{W^{1, n}}^{n} \leq 1\right\}} \supset \overline{\left\{J u:\|u\|_{W^{1, n}}^{n} \leq C\|J u\|_{\mathcal{H}^{1}} \leq M\right.}\right\}
$$

contains a ball. It follows that $\overline{\mathrm{co}}(V)$ contains a ball centered at the origin. By Lemma 3.1, $\left\{\sum_{j=1}^{\infty} M \lambda^{j} J u^{j}:\left\|u^{j}\right\|_{W^{1, n}} \leq 1\right.$ for every $\left.j \in \mathbb{N}, \sum_{j=1}^{\infty}\left|\lambda^{j}\right|<\infty\right\}$ is not of the first category, contradicting Theorem 1.2 .

For the second claim recall that $J\left(W^{1, n}\left(\mathbb{R}^{n}, \mathbb{R}^{n}\right)\right)$ is dense in $\mathcal{H}^{1}\left(\mathbb{R}^{n}\right)$. The claim follows from the first part of the proposition.

Note that the proofs of Theorem 1.2 and Proposition 3.2 only use (implicitly) the infinity behavior of mappings in $W^{1, n}\left(\mathbb{R}^{n}, \mathbb{R}^{n}\right)$ and do not put restraints on the local behavior of solutions of the Jacobian equation. In particular, the arguments do not apply in bounded domains or to mappings in $\dot{W}^{1, n}\left(\mathbb{R}^{n}, \mathbb{R}^{n}\right)$.

\section{Theorem 1.4 AND ITS VARIANTS}

The equivalence between conditions (i)-(ii) of Theorem 1.4 is proved in the case $A_{1}=0$ in 4.1 , and the case $A_{1} \in\{\operatorname{div}$, curl $\}$ is covered in 4.2 . The nonsurjectivity claim of Theorem 1.4 is shown in 44.3 . In 4.4 we present another equivalent condition in the case $A_{1}=A_{2}=0$, and an analogue of Theorem 1.4 is discussed in 4.5.

4.1. Proof of equivalence (i) $\Leftrightarrow$ (ii) when $A_{1}=0$. We start by explaining the main ideas behind the proof of implication (i) $\Rightarrow$ (ii) when $A_{1}=0$. The converse is, of course, classical. The argument is based loosely on P. Strzelecki's proof of [42, Theorem 2.1] (Corollary 5.10 in this article). 
We fix $x \in \mathbb{R}^{n}$ and $t>0$ aspiring to estimate

$$
\eta_{t} * \mathscr{B}(u, v)(x)=\sum_{i=1}^{m_{1}} \sum_{j=1}^{m_{2}} \sum_{|\alpha| \leq k_{1},|\beta| \leq k_{2}} c_{i, j, \alpha, \beta} \int_{\mathbb{R}^{n}} \eta_{t}(x-\cdot) \partial^{\alpha} u_{i} \partial^{\beta} v_{j} .
$$

As in e.g. 44 and 42, given $i, j, \alpha, \beta$ we wish to move some orders of differentiation from $u_{i}$ onto the test function $\eta_{t}(x-\cdot)$. To this end, we use the Leibniz rule to write $\partial^{\alpha}\left(\eta_{t}(x-\cdot) u_{i}\right)=\sum_{\gamma \leq \alpha}\left(\begin{array}{l}\alpha \\ \gamma\end{array}\right) \partial^{\alpha-\gamma}\left[\eta_{t}(x-\cdot)\right] \partial^{\gamma} u_{i}$ and assumption (ii) to get $\int_{\mathbb{R}^{n}} \mathscr{B}\left(\eta_{t}(x-\cdot) u, v\right)=0$. In the estimation of the integrals $\int_{\mathbb{R}^{n}} \partial^{\alpha-\gamma} \eta_{t}(x-$ .) $\partial^{\gamma} u_{i} \partial^{\beta} v_{j}$ we want to use the higher order Poincaré inequality, Lemma 2.3. on the factors $\partial^{\gamma} u_{i}$ in order to cancel the negative powers of $t$ that the differentiation of $\eta_{t}(x-\cdot)$ creates. In order for the Poincaré inequality to be applicable we need to subtract a suitable polynomial from $u$ before using the Leibniz rule.

At this point the obstacle is that even though given any polynomial $P: \mathbb{R}^{n} \rightarrow$ $\mathbb{R}^{m_{1}}$ we have $\int_{\mathbb{R}^{n}} \mathscr{B}\left(\eta_{t}(x-\cdot)(u-P), v\right)=0$, it is not guaranteed that the use of the Leibniz rule

$$
\partial^{\alpha}\left[\eta_{t}(x-\cdot)\left(u_{i}-P_{i}\right)\right]=\sum_{\gamma \leq \alpha}\left(\begin{array}{l}
\alpha \\
\gamma
\end{array}\right) \partial^{\alpha-\gamma}\left[\eta_{t}(x-\cdot)\right] \partial^{\gamma}\left(u_{i}-P_{i}\right)
$$

is successful in every term. Indeed, if $|\alpha|>\operatorname{deg} P_{i}+1$, then $P_{i}$ is in general unsuitable for use in the Poincaré inequality when $|\gamma|=|\alpha|-1$, since $\partial^{\gamma} P_{i}=0$. On the other hand, if $|\alpha| \leq \operatorname{deg} P_{i}$, then the term with $\gamma=\alpha$ produces unwanted extra terms.

As a consequence, we first need to manipulate $\mathscr{B}$ in such a way that $|\alpha|$ can be taken to be the same in every term of $\mathscr{B}$ (and the degree of $P$ is $|\alpha|-1$ ). We accomplish this in Lemmas 4.1 and 4.2. and thereby the higher order Poincaré inequality becomes available.

Lemma 4.1. Suppose $\mathscr{B}$ satisfies condition (i) of Theorem 1.4 Then, for every $l \leq k_{1}+k_{2}$ and every $(u, v) \in W^{k_{1}, p_{1}}\left(\mathbb{R}^{n}, \mathbb{R}^{m_{1}}\right) \times W_{A_{2}}^{k_{2}, p_{2}}\left(\mathbb{R}^{n}, \mathbb{R}^{m_{2}}\right)$, the integral of

$$
\mathscr{B}_{l}(u, v):=\sum_{i, j} \sum_{|\alpha+\beta|=l} c_{i, j, \alpha, \beta} \partial^{\alpha} u_{i} \partial^{\beta} v_{j}
$$

vanishes. Furthermore, $\mathscr{B}_{0}=0$.

Proof. Let $(u, v) \in W^{k_{1}, p_{1}}\left(\mathbb{R}^{n}, \mathbb{R}^{m_{1}}\right) \times W_{A_{2}}^{k_{2}, p_{2}}\left(\mathbb{R}^{n}, \mathbb{R}^{m_{2}}\right)$. When $\tau>0$, note that $A_{2}\left[(v(\tau \cdot)]=\tau^{q} A_{2} v(\tau \cdot)=0\right.$. Thus, by the assumption on $\mathscr{B}$ and a change of variables,

$$
0=\int_{\mathbb{R}^{n}} \mathscr{B}(u(\tau \cdot), v(\tau \cdot))=\tau^{-n} \sum_{l=0}^{k_{1}+k_{2}} \tau^{l} \int_{\mathbb{R}^{n}} \mathscr{B}_{l}(u, v) .
$$

By letting $\tau$ vary we get $\int_{\mathbb{R}^{n}} \mathscr{B}_{l}(u, v)=0$ for every $l$.

Since the integral $\int_{\mathbb{R}^{n}} \mathscr{B}_{0}(u, v)=\sum_{i, j} c_{i, j, 0,0} \int_{\mathbb{R}^{n}} u_{i} v_{j}$ vanishes for every $(u, v) \in$ $W^{k_{1}, p_{1}}\left(\mathbb{R}^{n}, \mathbb{R}^{m_{1}}\right) \times W_{A_{2}}^{k_{2}, p_{2}}\left(\mathbb{R}^{n}, \mathbb{R}^{m_{2}}\right)$, we conclude that $\sum_{j} c_{i, j, 0,0} v_{j}=0$ for every $i \in\left\{1, \ldots, m_{1}\right\}$ and every $v \in W_{A_{2}}^{k_{2}, p_{2}}\left(\mathbb{R}^{n}, \mathbb{R}^{m_{2}}\right)$, and the claim $\mathscr{B}_{0}=0$ follows.

We now use (4.1) to write $\mathscr{B}=\sum_{l=1}^{k_{1}+k_{2}} \mathscr{B}_{l}$ and treat the operators $\mathscr{B}_{l}$ separately. The following lemma allows us to further decompose the operators $\mathscr{B}_{l}$ so that in each new operator the value $|\alpha|$ is the same in every term. 
Lemma 4.2. Suppose $\mathscr{B}$ satisfies condition (i) of Theorem 1.4. Then we may write $\mathscr{B}=\sum_{l_{1}=1}^{k_{1}} \sum_{l_{2}=0}^{k_{2}} \mathscr{B}_{l_{1}, l_{2}}+R$, where for every $l_{1} \in\left\{1, \ldots, k_{1}\right\}, l_{2} \in\left\{0, \ldots, k_{2}\right\}$, and $(u, v) \in W^{k_{1}, p_{1}}\left(\mathbb{R}^{n}, \mathbb{R}^{m_{1}}\right) \times W_{A_{2}}^{k_{2}, p_{2}}\left(\mathbb{R}^{n}, \mathbb{R}^{m_{2}}\right)$ the bilinear quantity

$$
\mathscr{B}_{l_{1}, l_{2}}(u, v):=\sum_{i, j} \sum_{|\alpha|=l_{1}} \sum_{|\beta|=l_{2}} \tilde{c}_{i, j, \alpha, \beta} \partial^{\alpha} u_{i} \partial^{\beta} v_{j}
$$

satisfies $\int_{\mathbb{R}^{n}} \mathscr{B}_{l_{1}, l_{2}}(u, v)=0$ and $\|R(u, v)\|_{\mathcal{H}^{1}} \lesssim\|u\|_{W^{k_{1}, p_{1}}}\|v\|_{W^{k_{2}, p_{2}}}$. Furthermore, if $c_{i, j, 0, \beta}=0$ for all $i, j, \beta$, then $\|R(u, v)\|_{\mathcal{H}^{1}} \lesssim \sum_{l=1}^{k_{1}}\left\|\nabla^{l} u\right\|_{L^{p_{1}}}\|v\|_{W^{k_{2}, p_{2}}}$.

Proof. The idea of the proof is to show the following estimate: when $i \in\left\{1, \ldots, m_{1}\right\}$, $j \in\left\{1, \ldots, m_{2}\right\}, k \in\{1, \ldots, n\},|\gamma|<k_{1}$ and $|\theta|<k_{2}$, we have

$$
\left\|\partial_{k}\left(\partial^{\gamma} u_{i} \partial^{\theta} v_{j}\right)\right\|_{\mathcal{H}^{1}} \lesssim\left\|u_{i}\right\|_{W^{k_{1}, p_{1}}}\left\|v_{j}\right\|_{W^{k_{2}, p_{2}}} .
$$

Before proving (4.3) we note that if the sum defining $\mathscr{B}_{l}$ in (4.1) contains terms $c_{i, j, \alpha, \beta} \partial^{\alpha} u_{i} \partial^{\beta} v_{j}$ and $c_{i, j, \alpha^{\prime}, \beta^{\prime}} \partial^{\alpha^{\prime}} u_{i} \partial^{\beta^{\prime}} v_{j}$ with $|\alpha+\beta|=\left|\alpha^{\prime}+\beta^{\prime}\right|$ and $|\alpha|<\left|\alpha^{\prime}\right|$, we may use (4.3) to replace $c_{i, j, \alpha, \beta} \partial^{\alpha} u_{i} \partial^{\beta} v_{j}$ by $-c_{i, j, \alpha, \beta} \partial^{\alpha+e_{k}} u_{i} \partial^{\beta-e_{k}} v_{j}$ for some $k \in\{1, \ldots, n\}$ (and add $c_{i, j, \alpha, \beta} \partial_{k}\left(\partial^{\alpha} u_{i} \partial^{\beta-e_{k}} v_{j}\right)$ to $R$ ). The claim then follows by induction.

In order to prove (4.3) let $x \in \mathbb{R}^{n}$ and $t>0$. Choose a cube $Q \supset B(x, t)$ with $|Q| \lesssim_{n} t^{n}$. We fix $\left.\epsilon \in\right] 0, p_{2}-1\left[\right.$ choosing $\epsilon$ such that $1^{*}<\left(p_{2}-\epsilon\right)^{\prime}$ (and furthermore $\left(p_{2}-\epsilon\right)^{\prime}<p_{1}^{*}$ if $\left.p_{1}<n\right)$ and define $\delta>0$ by $\left(p_{1}-\delta\right)^{*}=\left(p_{2}-\epsilon\right)^{\prime}$. An integration by parts and Hölder's and Poincaré's inequalities yield

$$
\begin{aligned}
& \int_{\mathbb{R}^{n}} \eta_{t}(x-\cdot) \partial_{k}\left(\partial^{\gamma} u_{i} \partial^{\theta} v_{j}\right) \\
= & -\int_{Q} \partial_{k}\left[\eta_{t}(x-\cdot)\right]\left[\left(\partial^{\gamma} u_{i}-\left(\partial^{\gamma} u_{i}\right)_{Q}\right) \partial^{\theta} v_{j}+\left(\partial^{\gamma} u_{i}\right)_{Q}\left(\partial^{\theta} v_{j}-\left(\partial^{\theta} v_{j}\right)_{Q}\right)\right] \\
\lesssim & \frac{1}{t}\left(f_{Q}\left|\partial^{\gamma} u_{i}-\left(\partial^{\gamma} u_{i}\right)_{Q}\right|^{\left(p_{2}-\epsilon\right)^{\prime}}\right)^{\frac{1}{\left(p_{2}-\epsilon\right)}}\left(f_{Q}\left|\partial^{\theta} v_{j}\right|^{p_{2}-\epsilon}\right)^{\frac{1}{p_{2}-\epsilon}} \\
+ & \frac{1}{t}\left|\left(\partial^{\gamma} u_{i}\right)_{Q}\right| f_{Q}\left|\left(\partial^{\theta} v_{j}-\left(\partial^{\theta} v_{j}\right)_{Q}\right)\right| \\
\lesssim & \sum_{|\alpha| \leq k_{1}} M_{p_{1}-\delta}\left(\left|\partial^{\alpha} u_{i}\right|\right)(x) \sum_{|\beta| \leq k_{2}} M_{p_{2}-\epsilon}\left(\left|\partial^{\beta} v_{j}\right|\right)(x) .
\end{aligned}
$$

Now (4.3) follows by using Hölder's inequality and the Hardy-Littlewood Maximal Theorem.

The proof of direction (i) $\Rightarrow$ (ii) in the case $A_{1}=0$ will be completed by applying the following lemma. Note that Lemma 4.3 readily generalizes to multilinear operators; this fact is recorded in Theorem 4.5. In the formulation of Lemma 4.3 the assumptions and conclusion are in mismatch in order for the lemma to be usable in 4.2

Lemma 4.3. Suppose $l_{1} \in\left\{1, \ldots, k_{1}\right\}, l_{2} \in\left\{0, \ldots, k_{2}\right\}$ and $\mathscr{B}_{l_{1}, l_{2}}$ is of the form (4.2). If $\int_{\mathbb{R}^{n}} \mathscr{B}_{l_{1}, l_{2}}(u, v)=0$ for every $(u, v) \in W^{k_{1}, p_{1}}\left(\mathbb{R}^{n}, \mathbb{R}^{m_{1}}\right) \times W_{A_{2}}^{k_{2}, p_{2}}\left(\mathbb{R}^{n}, \mathbb{R}^{m_{2}}\right)$, then $\left\|\mathscr{B}_{l_{1}, l_{2}}(u, v)\right\|_{\mathcal{H}^{1}} \lesssim\left\|\nabla^{l_{1}} u\right\|_{L^{p_{1}}}\left\|\nabla^{l_{2}} v\right\|_{L^{p_{2}}}$ for every $(u, v) \in \dot{W}^{l_{1}, p_{1}}\left(\mathbb{R}^{n}, \mathbb{R}^{m_{1}}\right) \times$ $W_{A_{2}}^{k_{2}, p_{2}}\left(\mathbb{R}^{n}, \mathbb{R}^{m_{2}}\right)$.

Proof. Let $(u, v) \in \dot{W}^{l_{1}, p_{1}}\left(\mathbb{R}^{n}, \mathbb{R}^{m_{1}}\right) \times W_{A_{2}}^{k_{2}, p_{2}}\left(\mathbb{R}^{n}, \mathbb{R}^{m_{2}}\right)$ and fix $x \in \mathbb{R}^{n}$ and $t>0$. Select a cube $Q \supset B(x, t)$ with $|Q| \lesssim_{n}|B(x, t)|$ and use Lemma 2.3 to select a 
polynomial $P_{Q}^{l_{1}-1}$. By assumption $\int_{\mathbb{R}^{n}} \mathscr{B}_{l_{1}, l_{2}}\left(\eta_{t}\left(u-P_{Q}^{l_{1}-1} u\right), v\right)=0$, and so the Leibniz rule gives

$$
=\sum_{i, j} \sum_{|\alpha|=l_{1},|\beta|=l_{2}} \sum_{\gamma<\alpha} c_{i, j, \alpha, \beta, \gamma} \int_{\mathbb{R}^{n}} \partial^{\alpha-\gamma}\left[\eta_{t}(x-\cdot)\right] \partial^{\gamma}\left(u_{i}-P_{Q}^{l_{1}-1} u_{i}\right) \partial^{\beta} v_{j} .
$$

With $i, j, \alpha, \beta$ and $\gamma$ fixed, we choose again $\epsilon \in] 0, p_{2}-1\left[\right.$ such that $1^{*}<\left(p_{2}-\epsilon\right)^{\prime}$ (and $\left(p_{2}-\epsilon\right)^{\prime}<p_{1}^{*}$ if $\left.p_{1}<n\right)$ and define $\delta>0$ by $\left(p_{1}-\delta\right)^{*}=\left(p_{2}-\epsilon_{2}\right)^{\prime}$. By Hölder's inequality and Lemma 2.3 .

$$
\begin{aligned}
& \int_{\mathbb{R}^{n}} \partial^{\alpha-\gamma}\left[\eta_{t}(x-\cdot)\right] \partial^{\gamma}\left(u_{i}-P_{Q}^{l_{1}-1} u_{i}\right) \partial^{\beta} v_{j} \\
\lesssim & t^{-\alpha \alpha-\gamma \mid} f_{Q}\left|\partial^{\gamma}\left(u_{i}-P_{Q}^{l_{1}-1} u_{i}\right) \partial^{\beta} v_{j}\right| \\
\lesssim & t^{-|\alpha-\gamma|}\left(f_{Q}\left|\partial^{\gamma}\left(u_{i}-P_{Q}^{l_{1}-1} u_{i}\right)\right|^{\left(p_{2}-\epsilon\right)^{\prime}}\right)^{\frac{1}{\left(p_{2}-\epsilon\right)^{\prime}}}\left(f_{Q}\left|\partial^{\beta} v_{j}\right|^{p_{2}-\epsilon}\right)^{\frac{1}{p_{2}-\epsilon}} \\
\lesssim & M_{p_{1}-\delta}\left(\nabla^{l_{1}} u_{i}\right) M_{p_{2}-\epsilon}\left(\partial^{\beta} v_{j}\right) .
\end{aligned}
$$

The claimed estimate follows from Hölder's inequality and the Hardy-Littlewood Maximal Theorem.

We have now showed the equivalence of conditions (i) and (ii) of Theorem 1.4 in the case $A_{1}=0$. In the proof the identity $\int_{\mathbb{R}^{n}} \mathscr{B}\left(\eta_{t}(x-\cdot) u, v\right)=0$ allowed us to pass partial derivatives from $u$ onto $\eta_{t}(x-\cdot)$ by using the Leibniz rule. Such a technique is not available when $A_{1} \in\{\operatorname{div}$, curl $\}$, as it is not guaranteed that $\eta_{t}(x-\cdot) u \in W_{A_{1}}^{k_{1}, p_{1}}\left(\mathbb{R}^{n}, \mathbb{R}^{n}\right)$. We overcome this obstacle in $\$ 4.2$ by using potentials for divergence and curl.

4.2. The case $A_{1} \in\{$ div, curl $\}$. Direction (ii) $\Rightarrow$ (i) is classical as before, and the challenge is direction (i) $\Rightarrow$ (ii). Let $A_{1}=$ div; the case $A_{1}=$ curl is handled similarly.

Let $u \in W_{\text {div }}^{k_{1}, p_{1}}\left(\mathbb{R}^{n}, \mathbb{R}^{n}\right)$ and $v \in W_{A_{2}}^{k_{2}, p_{2}}\left(\mathbb{R}^{n}, \mathbb{R}^{n}\right)$. Using the potential $\Phi$ given by Proposition 2.10 and identifying $\mathbb{R}_{\Delta}^{n \times n}$ with $\mathbb{R}^{n(n-1) / 2}$ we write

$$
\begin{aligned}
\mathscr{B}(u, v) & =\sum_{i=1}^{n} \sum_{j=1}^{m_{2}} \sum_{|\alpha| \leq k_{1},|\beta| \leq k_{2}} c_{i, j, \alpha, \beta} \partial^{\alpha} u_{i} \partial^{\beta} v_{j} \\
& =\sum_{i=1}^{n(n-1) / 2} \sum_{j=1}^{m_{2}} \sum_{1 \leq|\alpha| \leq k_{1}+1,|\beta| \leq k_{2}} \tilde{c}_{i, j, \alpha, \beta} \partial^{\alpha} \Phi_{i} \partial^{\beta} v_{j} \\
& =: \quad \tilde{\mathscr{B}}(\Phi, v) .
\end{aligned}
$$

Using Lemmas 4.2 and 4.3 we may further decompose $\tilde{\mathscr{B}}=\sum_{l_{1}=1}^{k_{1}+1} \sum_{l_{2}=0}^{k_{2}} \tilde{\mathscr{B}}_{l_{1}, l_{2}}+R$, where

$$
\tilde{\mathscr{B}}_{l_{1}, l_{2}}(\Phi, v):=\sum_{i=1}^{n(n-1) / 2} \sum_{j=1}^{m_{2}} \sum_{|\alpha|=l_{1},|\beta|=l_{2}} \tilde{c}_{i, j, \alpha, \beta} \partial^{\alpha} \Phi_{i} \partial^{\beta} v_{j}
$$

for all $l_{1}, l_{2}$ and $\|R(\Phi, v)\|_{\mathcal{H}^{1}} \lesssim \sum_{l=1}^{k_{1}}\left\|\nabla^{l} \Phi\right\|_{L^{p_{1}}}\|v\|_{W^{k_{2}, p_{2}}}$. By Lemma 4.3 and Proposition 2.11.

$$
\|\tilde{\mathscr{B}}(\Phi, v)\|_{\mathcal{H}^{1}} \lesssim \sum_{l=1}^{k_{1}}\left\|\nabla^{l} \Phi\right\|_{L^{p_{1}}}\|v\|_{W^{k_{2}, p_{2}}} \lesssim\|u\|_{W^{k_{1}, p_{1}}}\|v\|_{W^{k_{2}, p_{2}}} .
$$


This completes the proof of implication (i) $\Rightarrow$ (ii) when $A_{1}=$ div.

4.3. Nonsurjectivity. We assume $A_{1}=$ curl, the case $A_{1}=\operatorname{div}$ being similar. The proof for $A_{1}=0$ is essentially a special case of the proof of Theorem 6.2. In the argument given below we tacitly assume that $A_{1} u^{l}=0$ and $A_{2} v^{l}=0$ for every $l \in \mathbb{N}$.

Completion of the proof of Theorem 1.4. Seeking contradiction, assume that the set

$$
\begin{aligned}
& \left\{\sum_{l=1}^{\infty} \lambda^{l} \mathscr{B}\left(u^{l}, v^{l}\right): \sup _{l \in \mathbb{N}}\left\|u^{l}\right\|_{W^{k_{1}, p_{1}}}\left\|v^{l}\right\|_{W^{k_{2}, p_{2}}} \leq 1, \sum_{l=1}^{\infty}\left|\lambda^{l}\right|<\infty\right\} \\
= & \left\{\sum_{l=1}^{\infty} \lambda^{l} \mathscr{B}\left(u^{l}, v^{l}\right): \sup _{l \in \mathbb{N}}\left(\left\|u^{l}\right\|_{W^{k_{1}, p_{1}}}^{2}+\left\|v^{l}\right\|_{W^{k_{2}, p_{2}}}^{2}\right) \leq 1, \sum_{l=1}^{\infty}\left|\lambda^{l}\right|<\infty\right\}
\end{aligned}
$$

is of the second category. Set $V:=\left\{\mathscr{B}(u, v):\|u\|_{W^{k_{1}, p_{1}}}^{2}+\|v\|_{W^{k_{2}, p_{2}}}^{2} \leq 1\right\}$. Now condition (iv) of Lemma 3.1 is satisfied.

As in the proof of Theorem 1.2. fix $b \in C_{c}^{\infty}\left(\mathbb{R}^{n}\right) \backslash\{0\}$. Use condition (i) of Lemma 3.1 to select, for every $\tau>0$, a mapping $(\tilde{u}, \tilde{v})=(u(\tau \cdot), v(\tau \cdot)) \in\left(W_{\text {curl }}^{k_{1}, p_{1}}\left(\mathbb{R}^{n}, \mathbb{R}^{n}\right) \times\right.$ $\left.W_{A_{2}}^{k_{2}, p_{2}}\left(\mathbb{R}^{n}, \mathbb{R}^{m_{2}}\right)\right) \backslash\{0\}$ satisfying

$$
\|b\|_{\mathrm{BMO}}=\|b(\tau \cdot)\|_{\mathrm{BMO}} \lesssim \frac{\int_{\mathbb{R}^{n}} b(\tau \cdot) \mathscr{B}(u(\tau \cdot), v(\tau \cdot))}{\|u(\tau \cdot)\|_{W^{k_{1}, p_{1}}}^{2}+\|v(\tau \cdot)\|_{W^{k_{2}, p_{2}}}^{2}} .
$$

We will show that (4.4) leads to a contradiction. We use Proposition 2.12 to write $u=\nabla \Psi$, where $\Psi \in \cap_{l=1}^{k_{1}+1} \dot{W}^{l, p_{1}}\left(\mathbb{R}^{n}\right)$, so that $\partial^{\alpha}\left[u_{i}(\tau \cdot)\right]=\tau^{-1} \partial^{\alpha+e_{i}}[\Psi(\tau \cdot)]$ for all $\alpha \in(\mathbb{N} \cup\{0\})^{n}$ and $i \in\{1, \ldots, n\}$. We choose a cube $Q \supset \operatorname{supp}(b)$ and assume, without loss of generality, that $\int_{Q} \Psi=0$.

We use the Leibniz rule (as before) and a change of variables to write

$$
\begin{aligned}
& \int_{\mathbb{R}^{n}} b(\tau \cdot) \mathscr{B}(u(\tau \cdot), v(\tau \cdot)) \\
= & \tau^{-1} \sum_{i, j} \sum_{\alpha, \beta} c_{i, j, \alpha, \beta} \int_{\mathbb{R}^{n}} b(\tau \cdot) \partial^{\alpha+e_{i}}[\Psi(\tau \cdot)] \partial^{\beta}\left[v_{j}(\tau \cdot)\right] \\
= & \tau^{-1} \sum_{i, j} \sum_{\alpha, \beta} \sum_{\gamma<\alpha+e_{i}} c_{i, j, \alpha, \beta, \gamma} \int_{\mathbb{R}^{n}} \partial^{\alpha+e_{i}-\gamma}[b(\tau \cdot)] \partial^{\gamma}[\Psi(\tau \cdot)] \partial^{\beta}\left[v_{j}(\tau \cdot)\right] \\
= & \sum_{i, j} \sum_{\alpha, \beta} \sum_{\gamma<\alpha+e_{i}} c_{i, j, \alpha, \beta, \gamma} \tau^{-n-1} \int_{\mathbb{R}^{n}} \tau^{\left|\alpha+e_{i}\right|} \partial^{\alpha+e_{i}-\gamma} b \partial^{\gamma} \Psi \tau^{|\beta|} \partial^{\beta} v_{j} \\
\lesssim & \sum_{i, j} \sum_{\alpha, \beta} \sum_{\gamma<\alpha+e_{i}} \tau^{-n-1} \int_{\mathbb{R}^{n}}\left|\tau^{\left|\alpha+e_{i}\right|} \partial^{\alpha+e_{i}-\gamma} b \partial^{\gamma} \Psi \tau^{|\beta|} \partial^{\beta} v_{j}\right| .
\end{aligned}
$$

We control single terms where $|\gamma| \geq 1$ by using Hölder's and Young's inequalities:

$$
\begin{array}{ll} 
& \tau^{-n-1} \int_{\mathbb{R}^{n}}\left|\tau^{\left|\alpha+e_{i}\right|} \partial^{\alpha+e_{i}-\gamma} b \partial^{\gamma} \Psi \tau^{|\beta|} \partial^{\beta} v_{j}\right| \\
\lesssim_{b} & \tau^{-1} M \tau^{\left|\alpha+e_{i}\right|-\frac{n}{p_{1}}}\left\|\partial^{\gamma} \Psi\right\|_{L^{p_{1}}} \frac{\tau^{|\beta|-\frac{n}{p_{2}}}}{M}\left\|\partial^{\beta} v_{j}\right\|_{L^{p_{2}}} \\
\leq & \tau^{-2} \frac{\left(M \tau^{\left|\alpha+e_{i}\right|-\frac{n}{p_{1}}}\right)^{2}}{2}\left\|\partial^{\gamma} \Psi\right\|_{L^{p_{1}}}^{2}+\frac{\left(\tau^{|\beta|-\frac{n}{p_{2}}}\right)^{2}}{2 M^{2}}\left\|\partial^{\beta} v_{j}\right\|_{L^{p_{2}}}^{2} .
\end{array}
$$


SAULI LINDBERG

The terms with $\gamma=0$ are, in turn, estimated by using the Poincaré inequality on $\Psi$ in $Q$ :

$$
\begin{aligned}
& \tau^{-n-1} \int_{\mathbb{R}^{n}}\left|\tau^{\left|\alpha+e_{i}\right|} \partial^{\alpha+e_{i}} b \Psi \tau^{|\beta|} \partial^{\beta} v_{j}\right| \\
\lesssim_{b} & \tau^{-1} M \tau^{\left|\alpha+e_{i}\right|-\frac{n}{p_{1}}}\|\Psi\|_{L^{p_{1}}(Q)} \frac{\tau^{|\beta|-\frac{n}{p_{2}}}}{M}\left\|\partial^{\beta} v_{j}\right\|_{L^{p_{2}}} \\
\lesssim_{b} & \tau^{-2} \frac{\left(M \tau^{\left|\alpha+e_{i}\right|-\frac{n}{p_{1}}}\right)^{2}}{2}\|\nabla \Psi\|_{L^{p_{1}(Q)}}^{2}+\frac{\left(\tau^{|\beta|-\frac{n}{p_{2}}}\right)^{2}}{2 M^{2}}\left\|\partial^{\beta} v_{j}\right\|_{L^{p_{2}}}^{2} .
\end{aligned}
$$

By combining the inequalities and using (4.4) we conclude that

$$
\begin{aligned}
& \sum_{i, j} \sum_{\alpha, \beta} \sum_{0<\gamma<\alpha+e_{i}}\left(\tau^{-2}\left(\tau^{p_{1}|\gamma|-n} \int_{\mathbb{R}^{n}}\left|\partial^{\gamma} \Psi\right|^{p_{1}}\right)^{\frac{2}{p_{1}}}\right. \\
& \left.+\left(\tau^{p_{2}|\beta|-n} \int_{\mathbb{R}^{n}}\left|\partial^{\beta} v_{j}\right|^{p_{2}}\right)^{\frac{2}{p_{2}}}\right) \\
& =\sum_{i, j} \sum_{\alpha, \beta} \sum_{0<\gamma<\alpha+e_{i}}\left(\tau^{-2}\left(\int_{\mathbb{R}^{n}}\left|\partial^{\gamma}[\Psi(\tau \cdot)]\right|^{p_{1}}\right)^{\frac{2}{p_{1}}}\right. \\
& \left.+\quad\left(\int_{\mathbb{R}^{n}}\left|\partial^{\beta}\left[v_{j}(\tau \cdot)\right]\right|^{p_{2}}\right)^{\frac{2}{p_{2}}}\right) \\
& \lesssim \tau^{-2} \sum_{l=1}^{k_{1}+1}\left\|\nabla^{l}[\Psi(\tau \cdot)]\right\|_{L^{p_{1}}}^{2}+\|v(\tau \cdot)\|_{W^{k_{2}, p_{2}}}^{2} \\
& \lesssim\|u(\tau \cdot)\|_{W^{k_{1}, p_{1}}}^{2}+\|v(\tau \cdot)\|_{W^{k_{2}, p_{2}}}^{2} \\
& \lesssim_{b} \int_{\mathbb{R}^{n}} b(\tau \cdot) \mathscr{B}(u(\tau \cdot), v(\tau \cdot)) \\
& \lesssim_{b} \sum_{i, j} \sum_{\alpha, \beta} \sum_{0<\gamma<\alpha+e_{i}}\left(\tau^{-2}\left(M^{p_{1}} \tau^{p_{1}\left|\alpha+e_{i}\right|-n} \int_{\mathbb{R}^{n}}\left|\partial^{\gamma} \Psi\right|^{p_{1}}\right)^{\frac{2}{p_{1}}}\right. \\
& \left.+\left(\frac{\tau^{p_{2}|\beta|-n}}{M^{p_{2}}} \int_{\mathbb{R}^{n}}\left|\partial^{\beta} v_{j}\right|^{p_{2}}\right)^{\frac{2}{p_{2}}}\right) .
\end{aligned}
$$

We get a contradiction by using the assumption $\left|\alpha+e_{i}\right|>|\gamma|$ for all multi-indices in the sums, choosing $M$ large enough and then choosing $\tau$ small enough.

4.4. A further equivalent condition in the case $A_{1}=A_{2}=0$. While condition (i) of Theorem 1.4tends to be easy to verify, it is not always completely transparent. In the following result we give, in the case $A_{1}=A_{2}=0$, another equivalent condition that can be tested by simple examination of the coefficients of $\mathscr{B}$.

Proposition 4.4. Suppose $\mathscr{B}$ satisfies the assumptions of Theorem 1.4 and $A_{1}=$ $A_{2}=0$. Then the following conditions are equivalent.

(i) For every $\gamma \in(\mathbb{N} \cup\{0\})^{n}$ and every $i \in\left\{1, \ldots, m_{1}\right\}$ and $j \in\left\{1, \ldots, m_{2}\right\}$,

$$
\sum_{\alpha+\beta=\gamma}(-1)^{|\alpha|} c_{i, j, \alpha, \beta}=0 .
$$

(ii) For every $(u, v) \in W^{k_{1}, p_{1}}\left(\mathbb{R}^{n}, \mathbb{R}^{m_{1}}\right) \times W^{k_{2}, p_{2}}\left(\mathbb{R}^{n}, \mathbb{R}^{m_{2}}\right)$,

$$
\|\mathscr{B}(u, v)\|_{\mathcal{H}^{1}} \lesssim\|u\|_{W^{k_{1}, p_{1}}}\|v\|_{W^{k_{2}, p_{2}}}
$$


Proof. When (i) holds and $(u, v) \in C_{c}^{\infty}\left(\mathbb{R}^{n}, \mathbb{R}^{m_{1}}\right) \times C_{c}^{\infty}\left(\mathbb{R}^{n}, \mathbb{R}^{m_{2}}\right)$, we compute

$$
\begin{aligned}
\int_{\mathbb{R}^{n}} \mathscr{B}(u, v) & =\sum_{\gamma} \sum_{i, j} \sum_{\alpha+\beta=\gamma} c_{i, j, \alpha, \beta} \int_{\mathbb{R}^{n}} \partial^{\alpha} u_{i} \partial^{\beta} v_{j} \\
& =\sum_{\gamma} \sum_{i, j}\left(\int_{\mathbb{R}^{n}} u_{i} \partial^{\gamma} v_{j} \sum_{\alpha+\beta=\gamma}(-1)^{|\alpha|} c_{i, j, \alpha, \beta}\right)=0 .
\end{aligned}
$$

By continuity, $\int_{\mathbb{R}^{n}} \mathscr{B}(u, v)=0$ for all $(u, v) \in W^{k_{1}, p_{1}}\left(\mathbb{R}^{n}, \mathbb{R}^{m_{1}}\right) \times W^{k_{2}, p_{2}}\left(\mathbb{R}^{n}, \mathbb{R}^{m_{2}}\right)$, and Theorem 1.4 then implies condition (ii).

For direction (ii) $\Rightarrow$ (i) we fix $\gamma \in(\mathbb{N} \backslash\{0\})^{n}$ and let $(u, v) \in C_{c}^{\infty}\left(\mathbb{R}^{n}, \mathbb{R}^{m_{1}+m_{2}}\right)$. By using an integration by parts we write, as above,

$$
0=\int_{\mathbb{R}^{n}} \mathscr{B}(u, v)=\sum_{i}\left(\sum_{j} \sum_{\gamma} \sum_{\alpha+\beta=\gamma}(-1)^{|\alpha|} c_{i, j, \alpha, \beta} \int_{\mathbb{R}^{n}} u_{i} \partial^{\gamma} v_{j}\right) .
$$

When $i_{0} \in\left\{1, \ldots, m_{1}\right\}$, letting $u_{i_{0}} \in C_{c}^{\infty}\left(\mathbb{R}^{n}\right)$ and $u_{i}=0$ for every $i \neq i_{0}$ we get $\sum_{j} \sum_{\gamma} \sum_{\alpha+\beta=\gamma}(-1)^{|\alpha|} c_{i_{0}, j, \alpha, \beta} \partial^{\gamma} v_{j}=0$ for all $v \in C_{c}^{\infty}\left(\mathbb{R}^{n}, \mathbb{R}^{m_{2}}\right)$. Fixing $\gamma \in$ $(\mathbb{N} \cup\{0\})^{n}$ with $|\gamma|=k_{1}+k_{2}$ and setting locally $v_{j_{0}}=x^{\gamma}$ and $v_{j}=0$ for all $j \neq j_{0}$ we obtain $\sum_{\alpha+\beta=\gamma}(-1)^{|\alpha|} c_{i_{0}, j_{0}, \alpha, \beta}=0$. Consequently,

$$
\sum_{i}\left(\sum_{j} \sum_{\gamma} \sum_{|\alpha+\beta|<l}(-1)^{|\alpha|} c_{i, j, \alpha, \beta} \int_{\mathbb{R}^{n}} u_{i} \partial^{\gamma} v_{j}\right)=0
$$

holds for $l=k_{1}+k_{2}$. Continuing by downward induction on $l$ we obtain condition (i).

4.5. A variant for homogeneous Sobolev spaces. The aim of this subsection is to give an analogue of Theorem 1.4 for homogeneous Sobolev spaces when $|\alpha|=k_{1}$ and $|\beta|=k_{2}$ in all non-vanishing terms of $\mathscr{B}$. This case is much easier than the one studied in Theorem 1.4 since the higher-order Poincaré inequality is readily available. Furthermore, multilinear operators are essentially as easy to handle as bilinear ones since Lemma 4.2 is not needed. We note in passing that terms satisfying $|\alpha|<k_{1}$ or $|\beta|<k_{2}$ can in some cases be treated in homogeneous Sobolev spaces by using Sobolev embeddings, but we do not pursue the matter here.

We consider multilinear operators $\mathscr{M}: \prod_{j=1}^{r} \dot{W}_{A_{j}}^{k_{j}, p_{j}}\left(\mathbb{R}^{n}, \mathbb{R}^{m_{j}}\right) \rightarrow L^{1}\left(\mathbb{R}^{n}\right)$ of the form

$$
\mathcal{M}\left(u^{1}, \ldots, u^{r}\right)=\sum_{\substack{i_{1} \in\left\{1, \ldots, m_{1}\right\} \\ \vdots \\ i_{r} \in\left\{1, \ldots, m_{r}\right\}}} \sum_{\left|\alpha_{1}\right|=k_{1}} c_{\alpha_{1}, \ldots, i_{r}, \alpha^{1}, \ldots, \alpha_{r}} \prod_{j=1}^{r} \partial^{\alpha^{j}} u_{i_{j}}^{j},
$$

where

$$
\left.k_{j} \geq 0, n \geq 2, m_{j} \geq 1, \text { and } p_{j} \in\right] 1, \infty\left[\text { with } \frac{1}{p_{1}}+\cdots+\frac{1}{p_{r}}=1 .\right.
$$

The case $A_{1}=\cdots=A_{r}=0$ of the following proposition follows e.g. from [15, Theorem 1] and the whole result seems to follow by using the ideas of [15] and the potentials for divergence and curl constructed in $\$ 2.5$. However, we feel that the 
explicit statement and fairly elementary proof of Theorem 4.5 bring further unity and clarity to the subject.

Theorem 4.5. Assume that (4.6) and (4.7) hold and $A_{1} \in\{0$, div, curl $\}$. The following conditions are equivalent.

(i) For every $\left(u^{1}, \ldots, u^{r}\right) \in \Pi_{j=1}^{r} \dot{W}_{A}^{k_{j}, p_{j}}\left(\mathbb{R}^{n}, \mathbb{R}^{m_{j}}\right)$,

$$
\int_{\mathbb{R}^{n}} \mathscr{M}\left(u^{1}, \ldots, u^{r}\right)=0 .
$$

(ii) For every $\left(u^{1}, \ldots, u^{r}\right) \in \Pi_{j=1}^{r} \dot{W}_{A_{j}}^{k_{j}, p_{j}}\left(\mathbb{R}^{n}, \mathbb{R}^{m_{j}}\right)$,

$$
\left\|\mathscr{M}\left(u^{1}, \ldots, u^{r}\right)\right\|_{\mathcal{H}^{1}} \lesssim \prod_{j=1}^{r}\left\|\nabla^{k_{j}} u^{j}\right\|_{L^{p_{j}}} .
$$

If $r=2$ and $A_{1}=A_{2}=0$, then (i) and (ii) are equivalent to the following condition:

(iii) For every $\gamma \in(\mathbb{N} \cup\{0\})^{n}$ and every $i \in\left\{1, \ldots, m_{1}\right\}$ and $j \in\left\{1, \ldots, m_{2}\right\}$,

$$
\sum_{\alpha+\beta=\gamma} c_{i, j, \alpha, \beta}=0 .
$$

In the case $A_{1}=0$ we note again that if $k_{1}=0$, then $\mathscr{M}=0$. The case $A_{1}=0$, $k_{1} \geq 1$ is proved by a simple adaptation of the proof of Lemma 4.3, and the case $A_{1} \in\{$ div, curl $\}$ is shown as in 4.2 . Furthermore, when $r=2$ and $A_{1}=A_{2}$, direction (i) $\Rightarrow$ (iii) follows from Proposition 4.4 while (iii) $\Rightarrow$ (i) checked by an integration by parts.

Remark 4.6. When $r \geq 3$, condition (iii) of Theorem4.5 has the following analogue: for every $\gamma \in(\mathbb{N} \cup\{0\})^{n}$, all indices $i_{j} \in\left\{1, \ldots, m_{j}\right\}$ and all multi-indices $\theta^{2}, \ldots, \theta^{r}$ with $\sum_{j=2}^{r} \theta^{j}=\gamma$,

$$
\sum_{\substack{\alpha^{1}+\ldots+\alpha^{r}=\gamma, \alpha^{1}+\alpha^{2} \geq \theta^{2}, \ldots, \alpha^{1}+\alpha^{r} \geq \theta^{r}}} c_{i_{1}, \ldots, i_{r}, \alpha^{1}, \ldots, \alpha^{r}} \prod_{j=2}^{r}\left(\begin{array}{c}
\alpha^{1} \\
\theta^{j}-\alpha^{j}
\end{array}\right)=0 .
$$

Condition (4.8) appears to be far too complicated to have practical use and so we omit the somewhat cumbersome proof of its equivalence to conditions (i) and (ii) of Theorem 4.5 .

\section{Applications to multilinear QUantities}

We present some corollaries of Theorems 1.4 and 4.5 and Proposition 4.4

5.1. Applications to classes of operators. We first use Theorem 1.4 and Proposition 4.4 to treat some general classes of bilinear partial differential operators. In the first result we study the product of two linear constant-coefficient partial differential operators. We define the operators $\mathscr{P}: W^{k_{1}, p_{1}}\left(\mathbb{R}^{n}, \mathbb{R}^{m_{1}}\right) \rightarrow L^{p_{1}}\left(\mathbb{R}^{n}\right)$ and $\mathcal{Q}: W^{k_{2}, p_{2}}\left(\mathbb{R}^{n}, \mathbb{R}^{m_{2}}\right) \rightarrow L^{p_{2}}\left(\mathbb{R}^{n}\right)$ by

$$
\mathscr{P}(u):=\sum_{i=1}^{m_{1}} \sum_{|\alpha| \leq k_{1}} c_{i, \alpha} \partial^{\alpha} u_{i} \quad \text { and } \quad \mathcal{Q}(v):=\sum_{j=1}^{m_{2}} \sum_{|\beta| \leq k_{2}} d_{j, \beta} \partial^{\beta} v_{j} .
$$

The estimate we present can be strengthened in an obvious manner when all the terms of $\mathscr{P}$ and/or $\mathcal{Q}$ have the same order. The result follows directly from Proposition 4.4 
ON THE HARDY SPACE THEORY OF COMPENSATED COMPACTNESS QUANTITIES 19

Corollary 5.1. Let $\mathscr{P}$ and $\mathcal{Q}$ be defined by (5.1). The following conditions are equivalent.

(i) $\|\mathscr{P}(u) \mathcal{Q}(v)\|_{\mathcal{H}^{1}} \lesssim\|u\|_{W^{k_{1}, p_{1}}}\|v\|_{W^{k_{2}, p_{2}}}$ for all $u \in W^{k_{1}, p_{1}}\left(\mathbb{R}^{n}, \mathbb{R}^{m}\right)$ and $v \in$ $W^{k_{2}, p_{2}}\left(\mathbb{R}^{n}, \mathbb{R}^{m}\right)$.

(ii) $\sum_{\alpha+\beta=\gamma}(-1)^{|\alpha|} c_{i, \alpha} d_{j, \beta}=0$ for all $i, j$ and all $\gamma \in(\mathbb{N} \cup\{0\})^{n}$.

In the following three corollaries we fix $n, k \in \mathbb{N}$, assume that $\left.p_{1}, p_{2} \in\right] 1, \infty[$ satisfy $1 / p_{1}+1 / p_{2}=1$ and define $\mathscr{P}: \mathcal{D}^{\prime}\left(\mathbb{R}^{n}\right) \rightarrow \mathcal{D}^{\prime}\left(\mathbb{R}^{n}\right)$ by

$$
\mathscr{P}(u):=\sum_{|\alpha| \leq k} c_{\alpha} \partial^{\alpha} u .
$$

Corollary 5.2. The following conditions are equivalent.

(i) $\|\mathscr{P}(u) v-\mathscr{P}(v) u\|_{\mathcal{H}^{1}} \lesssim\|u\|_{W^{k, p_{1}}}\|v\|_{W^{k, p_{2}}}$ for all $u \in W^{k, p_{1}}\left(\mathbb{R}^{n}\right)$ and $v \in$ $W^{k, p_{2}}\left(\mathbb{R}^{n}\right)$.

(ii) $|\alpha|$ is even in all the nonzero terms of $\mathscr{P}$.

Corollary 5.3. The following conditions are equivalent.

(i) $\|\mathscr{P}(u) v+\mathscr{P}(v) u\|_{\mathcal{H}^{1}} \lesssim\|u\|_{W^{k, p_{1}}}\|v\|_{W^{k, p_{2}}}$ for all $u \in W^{k, p_{1}}\left(\mathbb{R}^{n}\right)$ and $v \in$ $W^{k, p_{2}}\left(\mathbb{R}^{n}\right)$.

(ii) $|\alpha|$ is odd in all the nonzero terms of $\mathscr{P}$.

Corollary 5.4. The following conditions are equivalent.

(i) $\|\mathscr{P}(u v)\|_{\mathcal{H}^{1}} \lesssim\|u\|_{W^{k, p_{1}}}\|v\|_{W^{k, p_{2}}}$ for all $u \in W^{k, p_{1}}\left(\mathbb{R}^{n}\right)$ and $v \in W^{k, p_{2}}\left(\mathbb{R}^{n}\right)$.

(ii) $c_{0}=0$.

Proof of Corollaries 5.2 5.3. We write

$$
\mathscr{B}(u, v):=\mathscr{P}(u) v-\mathscr{P}(v) u=\sum_{|\alpha| \leq k} c_{\alpha}\left(v \partial^{\alpha} u-u \partial^{\alpha} v\right) .
$$

Condition (i) of Proposition 4.4 is satisfied if and only if $\left[(-1)^{|\gamma|}-1\right] c_{\gamma}=0$ for every $\gamma \in(\mathbb{N} \backslash\{0\})^{n}$, that is, if and only if condition (ii) of Corollary 5.2 holds. Proposition 4.4 now implies Corollary 5.2. The proof of Corollary 5.3 is almost identical.

Proof of Corollary 5.4. The direction (i) $\Rightarrow$ (ii) is obvious. For the other direction we verify condition (ii) of Theorem 1.4 by fixing $\alpha$ with $|\alpha| \geq 1$ and noting that $\int_{\mathbb{R}^{n}} \partial^{\alpha}(u v)=0$ for all $(u, v) \in W^{k, p_{1}}\left(\mathbb{R}^{n}\right) \times W^{k, p_{2}}\left(\mathbb{R}^{n}\right)$.

5.2. $\mathcal{H}^{1}$ regularity of specific operators. In this subsection we note that Theorem 4.5 directly implies $\mathcal{H}^{1}$ regularity with a natural norm estimate for many familiar compensated compactness quantities. In fact, the elementary cases $A_{1} \in$ $\{0$, curl $\}$ (which do not require the use of the somewhat technical potentials for divergence-free fields) suffice in every example presented here. Almost all of the results are from the original milestone paper [4].

Corollary 5.5 ( [4]). Suppose $p, q \in] 1, \infty[$ satisfy $1 / p+1 / q=1$ and let $v \in$ $L_{\text {div }}^{p}\left(\mathbb{R}^{n}, \mathbb{R}^{n}\right)$ and $u \in \dot{W}^{1, q}\left(\mathbb{R}^{n}\right)$. Then

$$
\|v \cdot \nabla u\|_{\mathcal{H}^{1}} \lesssim\|v\|_{L^{p}}\|\nabla u\|_{L^{q}} .
$$

Proof. Since $\int_{\mathbb{R}^{n}} v \cdot \nabla u=-\langle\operatorname{div} v, u\rangle=0$ for all $v \in L_{\mathrm{div}}^{p}\left(\mathbb{R}^{n}, \mathbb{R}^{n}\right)$ and $u \in C_{c}^{\infty}\left(\mathbb{R}^{n}\right)$, the claim follows from Theorem 4.5 and the density of $C_{c}^{\infty}\left(\mathbb{R}^{n}\right)$ in $\dot{W}^{1, q}\left(\mathbb{R}^{n}\right)$. 
Corollary 5.5 easily implies the $\mathcal{H}^{1}$ regularity of the div-curl quantity since $\nabla: \dot{W}^{1, q}\left(\mathbb{R}^{n}\right) \rightarrow L_{\text {curl }}^{q}\left(\mathbb{R}^{n}, \mathbb{R}^{n}\right)$ is a surjection.

Corollary 5.6 ( [4]). Suppose $E \in L_{\text {div }}^{p}\left(\mathbb{R}^{n}, \mathbb{R}^{n}\right)$ and $B \in L_{\text {curl }}^{q}\left(\mathbb{R}^{n}, \mathbb{R}^{n}\right)$, where $p, q \in] 1, \infty\left[\right.$ satisfy $1 / p+1 / q=1$. Then $\|E \cdot B\|_{\mathcal{H}^{1}} \lesssim\|E\|_{L^{p}}\|B\|_{L^{q}}$.

Since $C_{c}^{\infty}\left(\mathbb{R}^{n}, \mathbb{R}^{n}\right)$ is dense in $\dot{W}^{1,2}\left(\mathbb{R}^{n}, \mathbb{R}^{n}\right)$, the following result follows from Theorem 4.5 by an integration by parts.

Corollary 5.7 ( [4]). Let $u \in \dot{W}^{1,2}\left(\mathbb{R}^{n}, \mathbb{R}^{n}\right)$ and $v \in \dot{W}_{\text {div }}^{1,2}\left(\mathbb{R}^{n}, \mathbb{R}^{n}\right)$. Then we have $\left\|\sum_{i, j=1}^{n} \partial_{j} u_{i} \partial_{i} v_{j}\right\|_{\mathcal{H}^{1}} \lesssim\|\mathrm{D} u\|_{L^{2}}\|\mathrm{D} v\|_{L^{2}}$.

Corollaries 5.5 and 5.7 have, among others, the following consequence on Leray solutions of the Navier-Stokes equations, where the formula $-\Delta p=\sum_{i, j=1}^{3} \partial_{j} u_{i} \partial_{i} u_{j}$ is used.

Corollary 5.8 ( $[4])$. Assume that a velocity field $u \in L^{2}(] 0, \infty\left[; \dot{W}^{1,2}\left(\mathbb{R}^{3}, \mathbb{R}^{3}\right)\right) \cap$ $L^{\infty}(] 0, \infty\left[; L^{2}\left(\mathbb{R}^{3}, \mathbb{R}^{3}\right)\right)$ and a pressure $p \in \mathcal{D}^{\prime}(] 0, \infty\left[\times \mathbb{R}^{3}\right)$ satisfy the incompressible Navier-Stokes equations $\partial_{t} u+(u \cdot \nabla) u+\nabla p=\nu \Delta u$ and $\operatorname{div} u=0$ with viscosity $\nu>0$. The convective term $(u \cdot \nabla) u$ belongs to $L^{2}(] 0, \infty\left[; \mathcal{H}^{1}\left(\mathbb{R}^{3}\right)\right)$ and $\Delta p$ belongs to $L^{1}(] 0, \infty\left[; \mathcal{H}^{1}\left(\mathbb{R}^{3}\right)\right)$.

Theorem 2.1 is a direct corollary of Theorem 4.5 since

$$
\int_{\mathbb{R}^{n}} J u=\sum_{j=1}^{n}(-1)^{j} \int_{\mathbb{R}^{n}} \partial_{j}\left(u_{1} \frac{\partial\left(u_{2}, \ldots, u_{n}\right)}{\partial\left(x_{1}, \ldots, \hat{\left.x_{j}, x_{n}\right)}\right.}\right)=0
$$

for every $u \in C_{c}^{\infty}\left(\mathbb{R}^{n}, \mathbb{R}^{n}\right)$. We also mention the following consequence of Theorem 2.1 .

Corollary 5.9 ( [4). If $u \in \dot{W}^{2, n}\left(\mathbb{R}^{n}\right)$, then the Hessian $\mathbf{H} u:=\operatorname{det}[(\nabla \otimes \nabla) u]$ belongs to $\mathcal{H}^{1}\left(\mathbb{R}^{n}\right)$ and $\|\mathbf{H} u\|_{\mathcal{H}^{1}} \lesssim\left\|\nabla^{2} u\right\|_{L^{n}}^{n}$.

The following generalization of Corollary 5.5 by P. Strzelecki and its proof in 42] were among the main inspirations behind Theorem 1.4 and Theorem 4.5.

Corollary 5.10 ( [42]). Let $u \in \dot{W}^{k, p}\left(\mathbb{R}^{n}\right)$, where $k \geq 1$ and $1<p<\infty$. Suppose $E=\left(E_{\alpha}\right)_{|\alpha|=k} \in L^{p^{\prime}}\left(\mathbb{R}^{n}, \mathbb{R}^{k n}\right)$ satisfies $\nabla^{k} \cdot E=0$, that is, $\sum_{|\alpha|=k} \int_{\mathbb{R}^{n}} E_{\alpha} \partial^{\alpha} \varphi=0$ for all $\varphi \in C_{c}^{\infty}\left(\mathbb{R}^{n}\right)$. Then $\left\|\sum_{|\alpha|=k} E_{\alpha} \partial^{\alpha} u\right\|_{\mathcal{H}^{1}} \lesssim\|E\|_{L^{p^{\prime}}}\left\|\nabla^{k} u\right\|_{L^{p}}$.

The last compensated compactness quantity we mention appears in the von Kármán equations ( 4, p. 261], see also [3] and the references contained therein). Condition (iii) of Theorem 4.5 is clearly satisfied.

Corollary 5.11 ( 4]). When $(u, v) \in \dot{W}^{2,2}\left(\mathbb{R}^{2}, \mathbb{R}^{2}\right)$, the Monge-Ampère form $[u, v]:=u_{x x} v_{y y}+u_{y y} v_{x x}-2 u_{x y} v_{x y}$ satisfies $\|[u, v]\|_{\mathcal{H}^{1}} \lesssim\left\|\nabla^{2} u\right\|_{L^{2}}\left\|\nabla^{2} v\right\|_{L^{2}}$.

5.3. The equivalence between $\mathcal{H}^{1}$ regularity and weak sequential continuity. All the $\mathcal{H}^{1}$ regular quantities mentioned in $\$ 5.2$ (and numerous others that are studied in [4]) are compensated compactness quantities. Accordingly, the following problem is presented at 4, p. 267]:

"Roughly speaking, we have on one hand 'weakly continuous nonlinear quantities' and on the other hand 'nonlinear quantities' that belong to $\mathscr{H}^{1}$. A natural - but vague - question is then to determine whether these two classes coincide." 
Coifman, Lions, Meyer and Semmes went on to point out that it is not clear how one should formulate precisely this very general question. Partial results are found in [4, [5] and [15. In this section we show that Theorem 4.5] implies a positive result that applies to a large collection of familiar compensated fcompactness quantities. By using the potentials for divergence-free and curl-free fields constructed in 92.5 , the result can also be deduced from [15, Theorem 1]. A negative result appears in 86.2

Before stating the result we note that in this context the class of multilinear quantities studied in Theorem 4.5 is more natural than the one treated in Theorem 1.4. Indeed, if we allow $\mathscr{B}$ to contain lower order derivatives, then easy examples (such as $\mathscr{B}(u, v):=u \cdot v$ for $u, v \in W^{1,2}\left(\mathbb{R}^{n}, \mathbb{R}^{m}\right)$ ) show that weak sequential continuity does not imply $\mathcal{H}^{1}$ regularity.

Corollary 5.12. Suppose $\mathscr{M}$ is as in Theorem 4.5 and $A_{j} \in\{0$, div, curl $\}$ for every $j \in\{1, \ldots, r\}$. The following conditions are equivalent.

(i) $\int_{\mathbb{R}^{n}} \mathcal{M}(u)=0$ for every $u \in \Pi_{j=1}^{r} C_{c, A_{j}}^{\infty}\left(\mathbb{R}^{n}, \mathbb{R}^{m_{j}}\right)$.

(ii) For every $u \in \Pi_{j=1}^{r} \dot{W}_{A_{j}}^{k_{j}, p_{j}}\left(\mathbb{R}^{n}, \mathbb{R}^{m_{j}}\right)$,

$$
\|\mathcal{M}(u)\|_{\mathcal{H}^{1}} \lesssim \prod_{j=1}^{r}\left\|\nabla^{k_{j}} u^{j}\right\|_{L^{p_{j}}} .
$$

(iii) If the mappings $u, u^{1}, u^{2}, \cdots \in \Pi_{j=1}^{r} \dot{W}_{A_{j}}^{k_{j}, p_{j}}\left(\mathbb{R}^{n}, \mathbb{R}^{m_{j}}\right)$ satisfy $u^{l} \rightarrow u$ in $\Pi_{j=1}^{r} \dot{W}^{k_{j}, p_{j}}\left(\mathbb{R}^{n}, \mathbb{R}^{m_{j}}\right)$, then $\mathcal{M}\left(u^{l}\right) \rightarrow \mathcal{M}(u)$ in $\mathcal{D}^{\prime}\left(\mathbb{R}^{n}\right)$.

The equivalence of (i) and (ii) follows from Proposition 2.13 and Theorem 4.5 and thus it suffices to prove that (i) $\Leftrightarrow$ (iii). The result is essentially classical, but we sketch the idea of a proof for the convenience of the reader.

Proof of direction (i) $\Rightarrow$ (iii). Suppose (i) holds, and assume that the mappings $u, u^{1}, u^{2}, \ldots$ belong to $\Pi_{j=1}^{r} \dot{W}_{A_{j}}^{k_{j}, p_{j}}\left(\mathbb{R}^{n}, \mathbb{R}^{m_{j}}\right)$ and $u^{l} \rightarrow u$ in $\Pi_{j=1}^{r} \dot{W}^{k_{j}, p_{j}}\left(\mathbb{R}^{n}, \mathbb{R}^{m_{j}}\right)$. By the estimates and equivalence of weak convergences recorded in Propositions 2.102 .12 we may assume that $A_{1}=\cdots=A_{r}=0$. When $\varphi \in C_{c}^{\infty}\left(\mathbb{R}^{n}\right)$, we use telescoping summation to write

$$
\int_{\mathbb{R}^{n}} \varphi \mathscr{M}\left(u^{l}\right)-\int_{\mathbb{R}^{n}} \varphi \mathscr{M}(u)=\sum_{j=1}^{r} \int_{\mathbb{R}^{n}} \varphi \mathscr{M}\left(u^{l, 1}, \ldots, u^{l, j-1}, u^{l, j}-u^{j}, u^{j+1}, \ldots, u^{r}\right),
$$

and the proof is completed by using the Leibniz rule and the Rellich-Kondrachov theorem on every term.

The following argument has been adapted from [8, pp. 14-15].

Sketch of the proof of (iii) $\Rightarrow$ (i). Let $u \in \prod_{j=1}^{r} C_{c, A_{j}}^{\infty}\left(\mathbb{R}^{n}, \mathbb{R}^{m_{j}}\right)$; we aim to prove that $\int_{\mathbb{R}^{n}} \mathscr{M}(u)=0$. By scaling we may assume that $\left.\operatorname{supp}(u) \subset\right]-1,1\left[{ }^{n}\right.$. We denote the periodic extension of $u$ from $]-1,1\left[{ }^{n}\right.$ to the whole space $\mathbb{R}^{n}$ by $U$. When $l \in \mathbb{N}$, we define $u^{l} \in \prod_{j=1}^{r} C_{c, A_{j}}^{\infty}\left(\mathbb{R}^{n}, \mathbb{R}^{m_{j}}\right)$ by

$$
\left(u^{l, 1}(x), \ldots, u^{l, r}(x)\right):=\left(\frac{1}{l^{k_{1}}} \chi_{]-1,1\left[{ }^{n}\right.}(x) U^{1}(l x), \ldots, \frac{1}{l^{k_{r}}} \chi_{]-1,1\left[^{n}\right.}(x) U^{r}(l x)\right) .
$$


Now $\nabla^{k_{j}} u^{l, j} \rightarrow 0$ for every $j \in\{1, \ldots, r\}$, and so, by assumption, $\int_{\mathbb{R}^{n}} \mathscr{M}\left(u^{l}\right) \rightarrow 0$. On the other hand, a straightforward computation gives $\int_{\mathbb{R}^{n}} \mathscr{M}\left(u^{l}\right)=\int_{\mathbb{R}^{n}} \mathscr{M}(u)$ for every $l \in \mathbb{N}$, and therefore $\int_{\mathbb{R}^{n}} \mathscr{M}(u)=0$.

\section{The null Lagrangian COndition and $\mathcal{H}^{1}$ Regularity}

In this section we assume that $n, m, k \in \mathbb{N}$. Recall from $\$ 2.4$ that a null Lagrangian $\mathscr{L}: X(n, m, k) \rightarrow \mathbb{R}$ is a polynomial of some degree $r \in \mathbb{N}$ and that $\mathscr{L}$ maps $\dot{W}^{k, r}\left(\mathbb{R}^{n}, \mathbb{R}^{m}\right)$ into $\mathcal{H}^{1}\left(\mathbb{R}^{n}\right)$ with the natural norm bound $\left\|\mathscr{L}\left(\nabla^{k} u\right)\right\|_{\mathcal{H}^{1}} \lesssim$ $\left\|\nabla^{k} u\right\|_{L^{r}}^{r}$ if and only if $\mathscr{L}$ is $r$-homogeneous. Our main aim is to show that for homogeneous polynomials, $\mathcal{H}^{1}$ regularity is a strictly weaker condition than weak sequential continuity. This provides, in the present context, a negative answer to the question of Coifman, Lions, Meyer and Semmes quoted in 55.3 .

6.1. Characterization of $\mathcal{H}^{1}$ regularity for polynomials. We start by fixing notation. We denote multiples of multi-indices by capital greek letters and endow them with the following ordering.

Definition 6.1. When $\Gamma:=\left(\gamma^{1}, \ldots, \gamma^{r}\right), \Theta:=\left(\theta^{1}, \ldots, \theta^{r}\right) \in\left((\mathbb{N} \cup\{0\})^{n}\right)^{r}$, we say that $\Gamma \prec \Theta$ if $\gamma^{1} \leq \theta^{1}, \ldots, \gamma^{r} \leq \theta^{r}$ with strict inequality for at least one index $i \in\{1, \ldots, r\}$.

When $r \geq 2$, a general $r$-homogeneous polynomial $\mathscr{L}: X(n, m, k) \rightarrow \mathbb{R}$ is of the form

$$
\mathscr{L}\left(\nabla^{k} u\right):=\sum_{\nu \in\{1, \ldots, m\}^{r}} \sum_{\left|\theta_{1}\right|, \cdots,\left|\theta_{r}\right|=k} C_{\Theta, \nu} \prod_{i=1}^{r} \partial^{\theta^{i}} u_{\nu_{i}} .
$$

Theorem 6.2. Let $r \geq 2$. Suppose $\mathscr{L}: X(n, m, k) \rightarrow \mathbb{R}$ is an $r^{\text {th }}$ order polynomial. The following conditions are equivalent.

(i) $\left\|\mathscr{L}\left(\nabla^{k} u\right)\right\|_{\mathcal{H}^{1}} \lesssim\left\|\nabla^{k} u\right\|_{L^{r}}^{r}$ for all $u \in \dot{W}^{k, r}\left(\mathbb{R}^{n}, \mathbb{R}^{m}\right)$.

(ii) $\int_{\mathbb{R}^{n}} \mathscr{L}\left(\nabla^{k} u\right)=0$ for every $u \in C_{c}^{\infty}\left(\mathbb{R}^{n}, \mathbb{R}^{m}\right)$ and $\mathscr{L}$ is $r$-homogeneous.

When (i) and (ii) hold, the set

$$
\left\{\sum_{j=1}^{\infty} \lambda^{j} \mathscr{L}\left(\nabla^{k} u^{j}\right): \sup _{j \in \mathbb{N}}\left\|u^{j}\right\|_{W^{k, r}} \leq 1, \sum_{j=1}^{\infty}\left|\lambda^{j}\right|<\infty\right\}
$$

is of the first category in $\mathcal{H}^{1}\left(\mathbb{R}^{n}\right)$.

When (i) holds, the $r$-homogeneity of $\mathscr{L}$ follows from a standard scaling argument. The converse direction cannot be proved by simply adapting the proof of Theorem [1.4 since $\mathscr{L}$ may contain terms where partial derivatives of $u_{1}$ do not appear in any of the factors. For this reason, we fix $\psi \in C_{c}^{\infty}\left(\mathbb{R}^{n}\right)$ with $\operatorname{supp}(\psi) \subset B(0,1)$ and $\int_{\mathbb{R}^{n}} \psi^{r} \neq 0$ and set $\eta:=\psi^{r}$ in the definition of the norm $\|h\|_{\mathcal{H}^{1}}:=\left\|\sup _{t>0}\left|h * \eta_{t}\right|\right\|_{L^{1}}$.

Proof of direction (ii) $\Rightarrow$ (i). Let $u \in \dot{W}^{k, r}\left(\mathbb{R}^{n}, \mathbb{R}^{m}\right), x \in \mathbb{R}^{n}$ and $t>0$. Then $\psi_{t}(x-\cdot) u \in W^{k, r}\left(\mathbb{R}^{n}, \mathbb{R}^{m}\right)$ since $\psi_{t}(x-\cdot)$ is compactly supported. By (ii) and approximation,

$$
\int_{\mathbb{R}^{n}} \mathscr{L}\left(\nabla^{k}\left[\psi\left(\frac{x-\cdot}{t}\right) u\right]\right)=0
$$


Next, the Leibniz rule gives

$$
\begin{aligned}
& \mathscr{L}\left(\nabla^{k}\left[\psi\left(\frac{x-\cdot}{t}\right) u\right]\right) \\
= & \sum_{\Theta, \nu} C_{\Theta, \nu} \prod_{i=1}^{r} \partial^{\theta^{i}}\left[\psi\left(\frac{x-\cdot}{t}\right) u_{\nu_{i}}\right] \\
= & \eta\left(\frac{x-\cdot}{t}\right) \mathscr{L}\left(\nabla^{k} u\right)+\sum_{\Theta, \nu} \sum_{\Gamma \prec \Theta} C_{\Theta, \Gamma, \nu} \prod_{i=1}^{r} \partial^{\theta^{i}-\gamma^{i}}\left[\psi\left(\frac{x-\cdot}{t}\right)\right] \partial^{\gamma^{i}} u_{\nu_{i}} .
\end{aligned}
$$

As before, we pick a cube $Q \supset B(x, t)$ with $|Q| \lesssim_{n} t^{n}$ and use the equality above, Lemma 2.3 and (6.2) to write

$$
\begin{aligned}
& \int_{\mathbb{R}^{n}} \eta_{t}(x-\cdot) \mathscr{L}\left(\nabla^{k} u\right) \\
= & \sum_{\Theta, \nu} C_{\Theta, \nu} \frac{1}{t^{n}} \int_{Q} \prod_{i=1}^{r}\left[\psi\left(\frac{x-\cdot}{t}\right) \partial^{\theta^{i}}\left(u_{\nu_{i}}-P_{Q}^{k-1} u_{\nu_{i}}\right)\right] \\
= & \sum_{\Theta, \nu} \sum_{\Gamma \prec \Theta} C_{\Theta, \Gamma, \nu} \frac{1}{t^{n}} \int_{Q} \prod_{i=1}^{r} \partial^{\theta^{i}-\gamma^{i}}\left[\psi\left(\frac{x-\cdot}{t}\right)\right] \partial^{\gamma^{i}}\left(u_{\nu_{i}}-P_{Q}^{k-1} u_{\nu_{i}}\right) .
\end{aligned}
$$

Consider a single term of the sum, choose $j \in\{1, \ldots, r\}$ such that $\gamma^{j}<\theta^{j}$ and let $\epsilon>0$ be small. Then, by Hölder's inequality and Lemma 2.3.

$$
\begin{aligned}
& \left|\frac{1}{t^{n}} \int_{Q} \prod_{i=1}^{r} \partial^{\theta^{i}-\gamma^{i}}\left[\psi\left(\frac{x-\cdot}{t}\right)\right] \partial^{\gamma^{i}}\left(u_{\nu_{i}}-P_{Q}^{k-1} u_{\nu_{i}}\right)\right| \\
\lesssim & t^{-\left|\theta^{j}-\gamma^{j}\right|}\left(f_{Q}\left|\partial^{\gamma^{j}}\left(u_{\nu_{j}}-P_{Q}^{k-1} u_{\nu_{j}}\right)\right|^{\frac{r-\epsilon}{1-\epsilon}}\right)^{\frac{1-\epsilon}{r-\epsilon}} \\
\cdot & \prod_{i \neq j} t^{-\left|\theta^{i}-\gamma^{i}\right|}\left(f_{Q}\left|\partial^{\gamma^{i}}\left(u_{\nu_{i}}-P_{Q}^{k-1} u_{\nu_{i}}\right)\right|^{r-\epsilon}\right)^{\frac{1}{r-\epsilon}} \\
\lesssim & {\left[M_{r-\epsilon}\left(\nabla^{k} u\right)(x)\right]^{r} . }
\end{aligned}
$$

We conclude via Hardy-Littlewood Maximal Theorem as before.

Completion of the proof of Theorem 6.2. Seeking contradiction, assume

$$
\left\{\sum_{j=1}^{\infty} \lambda^{j} \mathscr{L}\left(\nabla^{k} u^{j}\right): \sup _{j \in \mathbb{N}}\left\|u^{j}\right\|_{W^{k, r}} \leq 1, \sum_{j=1}^{\infty}\left|\lambda^{j}\right|<\infty\right\}
$$

is of the second category in $\mathcal{H}^{1}\left(\mathbb{R}^{n}\right)$. Set $V:=\left\{ \pm \mathscr{L}\left(\nabla^{k} u\right):\|u\|_{W^{k, r}} \leq 1\right\} \subset$ $\mathcal{H}^{1}\left(\mathbb{R}^{n}\right)$; by condition (i) of Theorem 6.2, $V$ is bounded. Now condition (iv) of Lemma 3.1 is satisfied, and therefore

$$
\|b\|_{\mathrm{BMO}} \lesssim \sup _{h \in V} \int_{\mathbb{R}^{n}} b h=\sup _{u \neq 0} \frac{\left|\int b \mathscr{L}\left(\nabla^{k} u\right)\right|}{\|u\|_{W^{k, r}}^{r}}
$$

for all $b \in \mathrm{BMO}\left(\mathbb{R}^{n}\right)$. We will show that (6.3) leads to a contradiction.

When $b \in C_{c}^{\infty}\left(\mathbb{R}^{n}\right) \backslash\{0\}$, we use the Leibniz rule as in the proof above to get

$$
\int_{\mathbb{R}^{n}} b^{r} \mathscr{L}\left(\nabla^{k} u\right)=\sum_{\Theta, \nu} \sum_{\Gamma \prec \Theta} C_{\Theta, \Gamma, \nu} \int_{\mathbb{R}^{n}} \prod_{i=1}^{r} \partial^{\theta^{i}-\gamma^{i}} b \partial^{\gamma^{i}} u_{\nu_{i}} .
$$


As in the proof of Theorem 1.2. we fix $b \in C_{c}^{\infty}\left(\mathbb{R}^{n}\right) \backslash\{0\}$ and a parameter $\tau>0$ and use (6.3) to select $\tilde{u}=u(\tau \cdot) \in W^{k, r}\left(\mathbb{R}^{n}, \mathbb{R}^{m}\right) \backslash\{0\}$ satisfying

$$
\left\|b^{r}\right\|_{\mathrm{BMO}}=\left\|b^{r}(\tau \cdot)\right\|_{\mathrm{BMO}} \lesssim \frac{\mid \int_{\mathbb{R}^{n}} b^{r}(\tau \cdot) \mathscr{L}\left(\nabla^{k}[u(\tau \cdot]) \mid\right.}{\|u(\tau \cdot)\|_{W^{k, r}}^{r}} .
$$

By (6.4) and a change of variables,

$$
\begin{aligned}
& \int_{\mathbb{R}^{n}} b^{r}(\tau \cdot) \mathscr{L}\left(\nabla^{k}[u(\tau \cdot])\right. \\
= & \sum_{\Theta, \sigma} \sum_{\Gamma \prec \Theta} C_{\Theta, \Gamma, \nu} \int_{\mathbb{R}^{n}} \prod_{i=1}^{r} \partial^{\theta^{i}-\gamma^{i}}[b(\tau \cdot)] \partial^{\gamma^{i}}\left[u_{\nu_{i}}(\tau \cdot)\right] \\
= & \sum_{\Theta, \sigma} \sum_{\Gamma \prec \Theta} C_{\Theta, \Gamma, \nu} \tau^{-n} \int_{\mathbb{R}^{n}} \prod_{i=1}^{r} \tau^{\left|\theta^{i}\right|} \partial^{\theta^{i}-\gamma^{i}} b \partial^{\gamma^{i}} u_{\nu_{i}} \\
\lesssim_{b} & \sum_{\Theta, \sigma} \sum_{\Gamma \prec \Theta} \tau^{-n} \int_{\mathbb{R}^{n}}\left|\prod_{i=1}^{r} \tau^{\left|\theta^{i}\right|} \partial^{\gamma^{i}} u_{\nu_{i}}\right| .
\end{aligned}
$$

We next control a single term by the generalized Young's inequality. Choosing $j \in\{1, \ldots, r\}$ such that $\gamma_{j}<\theta_{j}$ we get

$$
\begin{aligned}
\int_{\mathbb{R}^{n}}\left|\prod_{i=1}^{r} \tau^{\left|\theta^{i}\right|} \partial^{\gamma^{i}} u_{\nu_{i}}\right| & =\int_{\mathbb{R}^{n}}\left|M \tau^{\left|\theta^{j}\right|} \partial^{\gamma^{j}} u_{\nu_{j}} \prod_{i \neq j} \frac{\tau^{\left|\theta^{i}\right|}}{M^{\frac{1}{r-1}}} \partial^{\gamma^{i}} u_{\nu_{i}}\right| \\
& \leq \frac{\tau^{r\left|\theta^{j}\right|} M^{r}}{r} \int_{\mathbb{R}^{n}}\left|\partial^{\gamma^{j}} u_{\nu_{j}}\right|^{r}+\sum_{i \neq j} \frac{\tau^{r\left|\theta^{i}\right|}}{r M^{r^{\prime}}} \int_{\mathbb{R}^{n}}\left|\partial^{\gamma^{i}} u_{\nu_{i}}\right|^{r} .
\end{aligned}
$$

By combining the inequalities and using (6.5) we conclude that

$$
\begin{aligned}
& \sum_{\Theta, \nu} \sum_{\Gamma \prec \Theta, \gamma^{j}<\theta^{j}}\left(\tau^{r\left|\gamma^{j}\right|-n} \int_{\mathbb{R}^{n}}\left|\partial^{\gamma^{j}} u_{\nu_{j}}\right|^{r}+\sum_{i \neq j} \tau^{r\left|\gamma^{i}\right|-n} \int_{\mathbb{R}^{n}}\left|\partial^{\gamma^{i}} u_{\nu^{i}}\right|^{r}\right) \\
= & \sum_{\Theta, \nu} \sum_{\Gamma \prec \Theta, \gamma^{j}<\theta^{j}}\left(\int_{\mathbb{R}^{n}} \mid \partial^{\gamma^{j}}\left[\left.u_{\nu_{j}}(\tau \cdot)\right|^{r}+\sum_{i \neq j} \int_{\mathbb{R}^{n}}\left|\partial^{\gamma^{i}}\left[u_{\nu_{i}}(\tau \cdot)\right]\right|^{r}\right)\right. \\
\lesssim & \|u(\tau \cdot)\|_{W^{k, r}}^{r} \\
\lesssim b & \sum_{\Theta, \nu} \sum_{\Gamma \prec \Theta, \gamma^{j}<\theta^{j}}\left(\tau^{r\left|\theta^{j}\right|-n} M^{r} \int_{\mathbb{R}^{n}}\left|\partial^{\gamma^{j}} u_{\nu_{j}}\right|^{r}+\sum_{i \neq j} \frac{\tau^{r\left|\theta^{i}\right|-n}}{M^{r^{\prime}}} \int_{\mathbb{R}^{n}}\left|\partial^{\gamma^{i}} u_{\nu_{i}}\right|^{r}\right) .
\end{aligned}
$$

For every term of the series we have $\left|\gamma^{j}\right|<\left|\theta^{j}\right|$ and $\left|\gamma^{i}\right| \leq\left|\theta^{i}\right|$ for all $i \neq j$, and once again we get a contradiction by choosing first $M$ large enough and then $\tau$ small enough.

6.2. $\mathcal{H}^{1}$ regular quantities not enjoying weak continuity. Theorem 6.2 allows us to find, for homogeneous polynomials of partial derivatives, a negative answer to the question of Coifman, Lions, Meyer, and Semmes quoted at $\$ 5.3$. There exist homogeneous polynomials $\mathscr{L}$ satisfying $\int_{\mathbb{R}^{n}} \mathscr{L}\left(\nabla^{k} \varphi\right)=0$ for all $\varphi \in C_{c}^{\infty}\left(\mathbb{R}^{n}, \mathbb{R}^{m}\right)$ but not being null Lagrangians. While this fact is undoubtedly well-known to experts, we give an explicit example for the reader's convenience. 
Proposition 6.3. Define $\mathscr{L}: X(3,2,2) \rightarrow \mathbb{R}$ via

$$
\begin{aligned}
\mathscr{L}\left(\nabla^{2}(u, v)\right) & :=\partial_{x x} u \partial_{y y} v \partial_{z z} v-\frac{1}{2} \partial_{x x} u \partial_{y z} v \partial_{y z} v-\partial_{x y} u \partial_{x y} v \partial_{z z} v \\
& +\partial_{y z} u \partial_{x x} v \partial_{y z} v-\partial_{z z} u \partial_{x x} v \partial_{y y} v+\frac{1}{2} \partial_{z z} u \partial_{x y} v \partial_{x y} v
\end{aligned}
$$

Then $\left\|\mathscr{L}\left(\nabla^{2}(u, v)\right)\right\|_{\mathcal{H}^{1}} \lesssim\left\|\nabla^{2}(u, v)\right\|_{L^{3}}^{3}$ for every $(u, v) \in \dot{W}^{2,3}\left(\mathbb{R}^{3}, \mathbb{R}^{2}\right)$ and yet $\left(u^{l}, v^{l}\right) \rightarrow(u, v)$ in $\dot{W}^{2,3}\left(\mathbb{R}^{3}, \mathbb{R}^{2}\right)$ does not imply that $\mathscr{L}\left(\nabla^{2}\left(u^{l}, v^{l}\right)\right) \rightarrow \mathscr{L}\left(\nabla^{2}(u, v)\right)$ in $\mathcal{D}^{\prime}\left(\mathbb{R}^{3}\right)$.

Proof. When $u, v \in C_{c}^{\infty}\left(\mathbb{R}^{3}\right)$, an integration by parts gives

$$
\begin{aligned}
\int_{\mathbb{R}^{3}} \mathscr{L}\left(\nabla^{2}(u, v)\right) & =\sum_{|\alpha|,|\beta|,|\gamma|=2} c_{\alpha, \beta, \gamma} \int_{\mathbb{R}^{n}} \partial^{\alpha} u \partial^{\beta} v \partial^{\gamma} v \\
& =\sum_{\left|\beta^{\prime}\right|+\left|\gamma^{\prime}\right|=6} d_{\beta^{\prime}, \gamma^{\prime}} \int_{\mathbb{R}^{n}} u \partial^{\beta^{\prime}} v \partial^{\gamma^{\prime}} v=0
\end{aligned}
$$

since, as is easily checked, $d_{\beta^{\prime}, \gamma^{\prime}}=0$ for every $\beta^{\prime}$ and $\gamma^{\prime}$. By Theorem 6.2 $\left\|\mathscr{L}\left(\nabla^{2}(u, v)\right)\right\|_{\mathcal{H}^{1}} \lesssim\left\|\nabla^{2}(u, v)\right\|_{L^{3}}^{3}$ for all $(u, v) \in \dot{W}^{2,3}\left(\mathbb{R}^{3}, \mathbb{R}^{2}\right)$.

However, $\mathscr{L}$ is not weakly sequentially continuous. Indeed, consider $u=u^{l}:=$ $x^{2} \psi_{B}$, where $\psi$ is a smooth cutoff function for a ball $B \subset \mathbb{R}^{3}$, and $v^{l}(x, y, z):=$ $l^{-2} \sin (l y+l z) \psi_{B}$. Then $\left(u^{l}, v^{l}\right) \rightarrow(u, 0)$ in $\dot{W}^{2,3}\left(\mathbb{R}^{3}, \mathbb{R}^{2}\right)$ but $\mathscr{L}\left(\nabla^{2}\left(u^{l}, v^{l}\right)\right)=$ $\sin ^{2}(l y+l z)$ in $B$ and so $\mathscr{L}\left(\nabla^{2}\left(u^{l}, v^{l}\right)\right)$ does not converge to 0 in $\mathcal{D}^{\prime}\left(\mathbb{R}^{3}\right)$.

The basic idea of Proposition 6.3 is that the integration by parts creates cancellations of partial derivatives of $v$ but such cancellations are not present in integrals $\int_{\mathbb{R}^{3}} \varphi \mathscr{L}\left(\nabla^{2}(u, v)\right)$, where $\varphi \in C_{c}^{\infty}\left(\mathbb{R}^{3}\right)$.

\section{Open problems Related to Question 1.1}

In this section we discuss open questions on the surjectivity of the Jacobian operator in Hardy spaces. The core of all the non-surjectivity results of this article is the incompatibility of the scaling properties of inhomogeneous Sobolev spaces and the target space $\mathcal{H}^{1}\left(\mathbb{R}^{n}\right)$, and this particular obstacle to surjectivity is not present when homogeneous Sobolev spaces are used as domains of definition of the operators or the surjectivity question is treated in bounded domains.

7.1. Homogeneous Sobolev spaces. Coifman, Lions, Meyer, and Semmes deduced Theorem 2.2 by proving the norm equivalence

$$
\|b\|_{\mathrm{BMO}} \approx \sup \left\{\int_{\mathbb{R}^{n}} b J u: u \in W^{1, n}\left(\mathbb{R}^{n}, \mathbb{R}^{n}\right), \int_{\mathbb{R}^{n}}|\mathrm{D} u|^{n} \leq 1\right\}
$$

and using direction $(i) \Rightarrow($ iii $)$ of Lemma 3.1, In view of (7.1) it is natural to ask whether $J$ maps the inhomogeneous Sobolev space $\dot{W}^{1, n}\left(\mathbb{R}^{n}, \mathbb{R}^{n}\right)$ onto $\mathcal{H}^{1}\left(\mathbb{R}^{n}\right)$. Recall that when $1<p<\infty$, the Hardy space $\mathcal{H}^{p}\left(\mathbb{R}^{n}\right)$ coincides with $L^{p}\left(\mathbb{R}^{n}\right)$. The following conjecture was posed by T. Iwaniec in 1997.

Conjecture 7.1 ( 20]). For every $n \geq 2$ and every $p \in[1, \infty[$ the Jacobian operator $J: \dot{W}^{1, n p}\left(\mathbb{R}^{n}, \mathbb{R}^{n}\right) \rightarrow \mathcal{H}^{p}\left(\mathbb{R}^{n}\right)$ has a continuous right inverse.

In 29] the author made progress on Conjecture 7.1 in the case $n=2, p=1$ via calculus of variations and Banach space geometry. Recall the norm $\|\cdot\|_{\mathrm{BMO}_{\mathcal{S}}}$ 
defined in (2.6) and denote the dual norm of $\mathcal{H}^{1}(\mathbb{C})$ by $\|\cdot\|_{\mathcal{H}_{S}^{1}}$. By the definition of $\|\cdot\|_{\mathrm{BMO}_{\mathcal{S}}}$

$$
\|J u\|_{\mathcal{H}_{\mathcal{S}}^{1}}=\sup _{\substack{\left.b \in \mathrm{VMO}_{(\mathbb{C})}\right) \\\|b\|_{\mathrm{BMO} \mathcal{S}}=1}} \frac{\int_{\mathbb{C}} b J u}{\int_{\mathbb{C}}\left|u_{\bar{z}}\right|^{2}} \int_{\mathbb{C}}\left|u_{\bar{z}}\right|^{2} \leq \int_{\mathbb{C}}\left|u_{\bar{z}}\right|^{2}
$$

for all (non-constant) $u \in \dot{W}^{1,2}(\mathbb{C}, \mathbb{C})$ and the inequality is sharp.

There exists, however, a large set of datas $h \in \mathcal{H}^{1}\left(\mathbb{R}^{n}\right)$ for which the equation $J u=h$ has a solution satisfying $\int_{\mathbb{C}}\left|u_{\bar{z}}\right|^{2}=\|J u\|_{\mathcal{H}_{\mathcal{S}}^{1}}$ - in particular, the set is closed and contains all the extreme points of the unit ball of $\left(\mathcal{H}^{1}\left(\mathbb{R}^{n}\right),\|\cdot\|_{\mathcal{H}_{S}^{1}}\right)$ (see [29, Theorems $1.19 \& 1.27]$ ). We refer to [29] and the forthcoming article [28] for more information on the topic. The author feels that there is already enough evidence for the following conjecture which appears as a question in [29.

Conjecture 7.2. For every $h \in \mathcal{H}^{1}(\mathbb{C})$ there exists $u \in \dot{W}^{1,2}(\mathbb{C}, \mathbb{C})$ such that $J u=h$ and $\|J u\|_{\mathcal{H}_{S}^{1}}=\int_{\mathbb{C}}\left|u_{\bar{z}}\right|^{2}$.

A positive answer to Conjecture 7.1 would imply, among other things, a new characterization of the duality pairing of $b \in \operatorname{BMO}(\mathbb{C})$ and $h \in \mathcal{H}^{1}(\mathbb{C})$ as an $L^{2}$ inner product via formula (2.5):

$$
\int_{\mathbb{C}} b h=\int_{\mathbb{C}} f \overline{K_{b} f}
$$

where $|\mathcal{S} f|^{2}-|f|^{2}=J_{u}=h$. Conjecture 7.2 in turn would imply, for instance, that we would have precise control on the norms of all the mappings involved: $\int_{\mathbb{C}}|f|^{2}=\|h\|_{\mathcal{H}_{\mathcal{S}}^{1}}$ and $\left\|K_{b}\right\|_{L^{2} \rightarrow L^{2}}=\|b\|_{\mathrm{BMO}_{\mathcal{S}}}$.

7.2. Bounded strongly Lipschitz domains. When a domain $\Omega \subset \mathbb{R}^{n}$ is bounded and strongly Lipschitz, the estimate $\|J u\|_{\mathcal{H}_{z}^{1}(\Omega)} \lesssim_{\Omega} \prod_{j=1}^{n}\left\|\nabla u_{j}\right\|_{L^{n}}$ holds for all $u \in W_{0}^{1, n}\left(\Omega, \mathbb{R}^{n}\right)$. In 31] Z.J. Lou, S.Z. Yang, and D.J. Song asked, in analogy to Question 1.1, whether $J$ maps $W_{0}^{1,2}\left(\Omega, \mathbb{R}^{2}\right)$ onto $\mathcal{H}_{z}^{1}(\Omega)$.

The author gave a negative answer for all (nonempty) bounded Lipschitz domains of $\mathbb{R}^{2}$ in [29, Theorem 8.12] by using the theory of T. Iwaniec and V. Sverák on mappings of integrable distortion (see [24]). We pose the following conjecture which appears as a question in [29].

Conjecture 7.3. The Jacobian maps $W^{1,2}(\Omega) \times W_{0}^{1,2}(\Omega)$ onto $\mathcal{H}_{z}^{1}(\Omega)$.

In view of the theory of the Beurling transform on domains, Conjecture 7.3 is more natural than the one for $J: W_{0}^{1,2}\left(\Omega, \mathbb{R}^{2}\right) \rightarrow \mathcal{H}_{z}^{1}(\Omega)$. In higher dimensions the correct analogue of Conjecture 7.3 appears to be whether $J$ maps $W^{1, n}\left(\Omega, \mathbb{R}^{n-1}\right) \times$ $W_{0}^{1, n}(\Omega)$ onto $\mathcal{H}_{z}^{1}(\Omega)$.

7.3. $L^{p}$ spaces. We start the discussion on the Jacobian equation with data in $L^{p}\left(\mathbb{R}^{n}\right)$ by presenting the following analogue of Theorem 1.2.

Theorem 7.4. For every $n \geq 2$ and $p \in] 1, \infty[$, the set

$$
\left\{\sum_{j=1}^{\infty} \lambda^{j} J u^{j}:\left\|u^{j}\right\|_{W^{1, n p}} \leq 1 \text { for every } j \in \mathbb{N}, \sum_{j=1}^{\infty}\left|\lambda^{j}\right|<\infty\right\}
$$

is of the first category in $L^{p}\left(\mathbb{R}^{n}\right)$. 
Theorem 7.4 is demonstrated by a proof similar to that of Theorem 1.2 by using the fact that $\|b(\tau \cdot)\|_{L^{p^{\prime}}}=\tau^{-n / p^{\prime}}\|b\|_{L^{p^{\prime}}}$ for all $b \in L^{p^{\prime}}\left(\mathbb{R}^{n}\right)$ and all $\tau>0$. The only notable difference is the use of the integration by parts - in this instance we make a further application of Hölder's inequality and estimate

$$
\begin{aligned}
\int_{\mathbb{R}^{n}} b J u & \leq \int_{\operatorname{supp}(b)}\left(M^{n}|u|^{n}+\frac{C(b)}{M^{n^{\prime}}}|\mathrm{D} u|^{n}\right) \\
& \leq C(b, p)\left(M^{n p} \int_{\mathbb{R}^{n}}|u|^{n p}+\frac{1}{M^{n^{\prime} p}} \int_{\mathbb{R}^{n}}|\mathrm{D} u|^{n p}\right)^{\frac{1}{p}} .
\end{aligned}
$$

The rest of the details are left to the reader.

An important step towards solving Conjecture 7.1 for $p \in] 1, \infty[$ would be to find out whether the following analogue of (7.1) holds:

$$
\|b\|_{L^{p^{\prime}}} \approx \sup _{\|\mathrm{D} u\|_{L^{n p}} \leq 1} \int_{\mathbb{R}^{n}} b J u \quad \text { for all } b \in L^{p^{\prime}}\left(\mathbb{R}^{n}\right) .
$$

Condition (7.2) is necessary for surjectivity of $J: \dot{W}^{1, n p}\left(\mathbb{R}^{n}, \mathbb{R}^{n}\right) \rightarrow L^{p}\left(\mathbb{R}^{n}\right)$ : setting $V:=\left\{J u:\|\mathrm{D} u\|_{L^{n p}} \leq 1\right\} \subset L^{p}\left(\mathbb{R}^{n}\right)$ we deduce from Lemma 3.1 that $\left\{\sum_{j=1}^{\infty} \lambda^{j} J u^{j}:\left\|\mathrm{D} u^{j}\right\|_{L^{n p}} \leq 1\right.$ for every $\left.j \in \mathbb{N}, \sum_{j=1}^{\infty}\left|\lambda^{j}\right|<\infty\right\}$ is of the second category in $L^{p}\left(\mathbb{R}^{n}\right)$ if and only if (7.2) holds. Conversely, if (7.2) holds, then many techniques of 29 formulated for $\mathcal{H}^{1}(\mathbb{C})$ are available in $L^{p}\left(\mathbb{R}^{n}\right)$, especially in the planar case.

When $n=2$, condition (7.2) has an interesting connection to operator norms of commutators $\mathcal{S} b-b \mathcal{S}$. Indeed, using formula (2.5) we get

$$
\sup _{\|\mathrm{D} u\|_{L^{n p} \leq 1}} \int_{\mathbb{R}^{n}} b J u \approx \sup _{\|f\|_{L^{2 p} \leq 1}} \int_{\mathbb{C}} f \overline{K_{b} f} \approx\|\mathcal{S} b-b \mathcal{S}\|_{L^{2 p} \rightarrow L^{(2 p)^{\prime}}}
$$

for every $b \in L^{p^{\prime}}(\mathbb{C})$, where the last two-sided estimate is proved by adapting the proof of equality of the operator norm and numerical radius of a self-adjoint operator in a Hilbert space. The author has been unable to prove or disprove (7.2).

\section{ACKNOWLEDGEMENT}

The author wishes to thank Kari Astala and Tadeusz Iwaniec for useful discussions on the subject matter of the article.

\section{REFERENCES}

[1] Kari Astala, Tadeusz Iwaniec, and Gaven Martin, Elliptic partial differential equations and quasiconformal mappings in the plane, Princeton Mathematical Series, vol. 48, Princeton University Press, Princeton, NJ, 2009.

[2] J. M. Ball, J. C. Currie, and P. J. Olver, Null Lagrangians, weak continuity, and variational problems of arbitrary order, J. Funct. Anal. 41 (1981), no. 2, 135-174.

[3] Pascal Cherrier and Albert Milani, Evolution equations of von Karman type, Lecture Notes of the Unione Matematica Italiana, vol. 17, Springer, Cham, 2015.

[4] R. Coifman, P.-L. Lions, Y. Meyer, and S. Semmes, Compensated compactness and Hardy spaces, J. Math. Pures Appl. (9) 72 (1993), no. 3, 247-286.

[5] Ronald R. Coifman and Loukas Grafakos, Hardy space estimates for multilinear operators. I, Rev. Mat. Iberoamericana 8 (1992), no. 1, 45-67.

[6] Ronald R. Coifman and Guido Weiss, Extensions of Hardy spaces and their use in analysis, Bull. Amer. Math. Soc. 83 (1977), no. 4, 569-645.

[7] G. Cupini, Bernard Dacorogna, and O. Kneuss, On the equation $\operatorname{det} \nabla u=f$ with no sign hypothesis, Calc. Var. Partial Differential Equations 36 (2009), no. 2, 251-283. 
[8] Bernard Dacorogna, Weak continuity and weak lower semicontinuity of nonlinear functionals, Lecture Notes in Mathematics, vol. 922, Springer-Verlag, Berlin-New York, 1982.

[9] Constantine M. Dafermos, Hyperbolic conservation laws in continuum physics, third ed., Grundlehren der Mathematischen Wissenschaften [Fundamental Principles of Mathematical Sciences], vol. 325, Springer-Verlag, Berlin, 2010.

[10] Ronald J. DiPerna, Compensated compactness and general systems of conservation laws, Trans. Amer. Math. Soc. 292 (1985), no. 2, 383-420.

[11] Lawrence C. Evans, Weak convergence methods for nonlinear partial differential equations, CBMS Regional Conference Series in Mathematics, vol. 74, Published for the Conference Board of the Mathematical Sciences, Washington, DC; by the American Mathematical Society, Providence, RI, 1990.

[12] C. Fefferman and E. M. Stein, $H^{p}$ spaces of several variables, Acta Math. 129 (1972), no. 3-4, 137-193.

[13] G. P. Galdi, An introduction to the mathematical theory of the Navier-Stokes equations, second ed., Springer Monographs in Mathematics, Springer, New York, 2011, Steady-state problems.

[14] Mariano Giaquinta and Luca Martinazzi, An introduction to the regularity theory for elliptic systems, harmonic maps and minimal graphs, second ed., Appunti. Scuola Normale Superiore di Pisa (Nuova Serie) [Lecture Notes. Scuola Normale Superiore di Pisa (New Series)], vol. 11, Edizioni della Normale, Pisa, 2012.

[15] Loukas Grafakos, Hardy space estimates for multilinear operators. II, Rev. Mat. Iberoamericana 8 (1992), no. 1, 69-92.

[16] _ Classical and modern Fourier analysis, Pearson Education, Inc., Upper Saddle River, NJ, 2004.

[17] Frédéric Hélein, Harmonic maps, conservation laws and moving frames, second ed., Cambridge Tracts in Mathematics, vol. 150, Cambridge University Press, Cambridge, 2002, Translated from the 1996 French original, With a foreword by James Eells.

[18] Lars Hörmander, The analysis of linear partial differential operators. I, second ed., Springer Study Edition, Springer-Verlag, Berlin, 1990, Distribution theory and Fourier analysis.

[19] _ Lectures on nonlinear hyperbolic differential equations, Mathématiques \& Applications (Berlin) [Mathematics \& Applications], vol. 26, Springer-Verlag, Berlin, 1997.

[20] Tadeusz Iwaniec, Nonlinear commutators and Jacobians, Proceedings of the conference dedicated to Professor Miguel de Guzmán (El Escorial, 1996), vol. 3, 1997, pp. 775-796.

[21] _ Null Lagrangians, the art of integration by parts, The interaction of analysis and geometry, Contemp. Math., vol. 424, Amer. Math. Soc., Providence, RI, 2007, pp. 83-102.

[22] Tadeusz Iwaniec and Gaven Martin, Geometric function theory and non-linear analysis, Oxford Mathematical Monographs, The Clarendon Press, Oxford University Press, New York, 2001.

[23] Tadeusz Iwaniec and Jani Onninen, $\mathcal{H}^{1}$-estimates of Jacobians by subdeterminants, Math. Ann. 324 (2002), no. 2, 341-358.

[24] Tadeusz Iwaniec and Vladimír Šverák, On mappings with integrable dilatation, Proc. Amer. Math. Soc. 118 (1993), no. 1, 181-188.

[25] Olivier Kneuss, On the equation $\operatorname{det} \nabla \phi=f$ prescribing $\phi=0$ on the boundary, Differential Integral Equations 25 (2012), no. 11-12, 1037-1052.

[26] Hideo Kozono and Hermann Sohr, New a priori estimates for the Stokes equations in exterior domains, Indiana Univ. Math. J. 40 (1991), no. 1, 1-27.

[27] Enno Lenzmann and Armin Schikorra, Sharp commutator estimates via harmonic extensions, arXiv:1609.08547 (2016).

[28] Sauli Lindberg, A note on the Jacobian equation in the plane, manuscript in preparation.

[29] - On the Jacobian equation and the Hardy space $\mathscr{H}^{1}(\mathbb{C})$, Ann. Acad. Sci. Fenn. Math. Diss. (2015), no. 160, Dissertation, University of Helsinki, Helsinki, 2015.

[30] Pierre-Louis Lions, Mathematical topics in fluid mechanics. Vol. 1, Oxford Lecture Series in Mathematics and its Applications, vol. 3, The Clarendon Press, Oxford University Press, New York, 1996, Incompressible models, Oxford Science Publications.

[31] Zeng Jian Lou, Shou Zhi Yang, and Dao Jin Song, Decompositions of the Hardy space $\mathscr{H}_{z}^{1}(\Omega)$, Acta Math. Sin. (Engl. Ser.) 21 (2005), no. 4, 949-954. 
[32] Yunguang Lu, Hyperbolic conservation laws and the compensated compactness method, Chapman \& Hall/CRC Monographs and Surveys in Pure and Applied Mathematics, vol. 128, Chapman \& Hall/CRC, Boca Raton, FL, 2003.

[33] S. Müller, Tang Qi, and B. S. Yan, On a new class of elastic deformations not allowing for cavitation, Ann. Inst. H. Poincaré Anal. Non Linéaire 11 (1994), no. 2, 217-243.

[34] Stefan Müller, A surprising higher integrability property of mappings with positive determinant, Bull. Amer. Math. Soc. (N.S.) 21 (1989), no. 2, 245-248.

[35] François Murat, Compacité par compensation, Ann. Scuola Norm. Sup. Pisa Cl. Sci. (4) 5 (1978), no. 3, 489-507.

[36] Compacité par compensation. II, Proceedings of the International Meeting on Recent Methods in Nonlinear Analysis (Rome, 1978), Pitagora, Bologna, 1979, pp. 245-256.

[37] P. J. Olver and J. Sivaloganathan, The structure of null Lagrangians, Nonlinearity 1 (1988), no. 2, 389-398.

[38] Peter J. Olver, Applications of Lie groups to differential equations, second ed., Graduate Texts in Mathematics, vol. 107, Springer-Verlag, New York, 1993.

[39] Martí Prats and Xavier Tolsa, A $T(P)$ theorem for Sobolev spaces on domains, J. Funct. Anal. 268 (2015), no. 10, 2946-2989.

[40] Hermann Sohr and Maria Specovius-Neugebauer, The Stokes problem for exterior domains in homogeneous Sobolev spaces, Theory of the Navier-Stokes equations, Ser. Adv. Math. Appl. Sci., vol. 47, World Sci. Publ., River Edge, NJ, 1998, pp. 185-205.

[41] Elias M. Stein, Harmonic analysis: real-variable methods, orthogonality, and oscillatory integrals, Princeton Mathematical Series, vol. 43, Princeton University Press, Princeton, NJ, 1993, With the assistance of Timothy S. Murphy, Monographs in Harmonic Analysis, III.

[42] Paweł Strzelecki, Hardy space estimates for higher-order differential operators, Indiana Univ. Math. J. 50 (2001), no. 3, 1447-1461.

[43] L. Tartar, Compensated compactness and applications to partial differential equations, Nonlinear analysis and mechanics: Heriot-Watt Symposium, Vol. IV, Res. Notes in Math., vol. 39, Pitman, Boston, Mass.-London, 1979, pp. 136-212.

[44] Luc Tartar, The compensated compactness method applied to systems of conservation laws, Systems of nonlinear partial differential equations (Oxford, 1982), NATO Adv. Sci. Inst. Ser. C Math. Phys. Sci., vol. 111, Reidel, Dordrecht, 1983, pp. 263-285.

[45] Henry C. Wente, An existence theorem for surfaces of constant mean curvature, J. Math. Anal. Appl. 26 (1969), 318-344.

Current address: Department of Mathematics, Universidad Autónoma de Madrid, E-28049 Madrid, Spain; ICMAT CSIC-UAM-UCM-UC3M, E-28049 Madrid, Spain

E-mail address: sauli.lindberg@uam.es 